Finite element simulation of gap opening between cladding tube and spacer grid in a fuel rod assembly using crystallographic models of irradiation growth and creep

\author{
Anirban Patra*, Carlos N. Tomé \\ Materials Science and Technology Division, Los Alamos National Laboratory, Los Alamos, NM \\ 87545 \\ * Corresponding author: \\ Anirban Patra \\ Materials Science and Technology Division \\ Los Alamos National Laboratory \\ Los Alamos, NM 87545, USA \\ Tel.: +1-505-665-4618 \\ Email: apatra@lanl.gov
}




\title{
Finite element simulation of gap opening between cladding tube and spacer grid in a fuel rod assembly using crystallographic models of irradiation growth and creep
}

\author{
Anirban Patra, Carlos N. Tomé \\ Materials Science and Technology Division, Los Alamos National Laboratory, Los Alamos, NM \\ 87545
}

\begin{abstract}
A physically-based crystal plasticity framework for modeling irradiation growth and creep is interfaced with the finite element code ABAQUS to study the contact forces and the gap evolution between the spacer grid and the cladding tube as a function of irradiation in a representative section of a fuel rod assembly. Deformation mechanisms governing the gap opening are identified and correlated to the texture-dependent material response. In the absence of coolant flow-induced vibrations, these simulations predict the contribution of irradiation growth and creep to the gap opening between the cladding tube and the springs and dimples on the spacer grid. The simulated contact forces on the springs and dimples are compared to available experimental and modeling data. Various combinations of external loads are applied on the springs and dimples to simulate fuel rods in the interior and at the periphery of the fuel rod assembly. It is found that loading conditions representative (to a first order approximation) of fuel rods at the periphery show higher gap opening. This is in agreement with in-reactor data, where rod leakages due to the synergistic effects of gap opening and coolant flow-induced vibrations were generally found to occur at the periphery of the fuel rod assembly.
\end{abstract}

\section{Introduction}

Grid-to-rod (GTR) fretting wear is a major concern for the design and operation of nuclear fuel rod assemblies in pressurized water reactors (PWRs) [1]-[4]. In a nuclear fuel rod assembly, the fuel rod (fuel pellets plus containing cladding tube) is supported by the spacer grid, which is a square pattern of blades with protruding springs and dimples that hold the cladding tube. Under reactor conditions, the fuel rod assembly is subject to significant doses of irradiation. This induces dimensional changes in the Zirconium alloy-based cladding tube and spacer grid due to irradiation growth and creep [5]. As a result of this irradiation-induced deformation, the springs and dimples may separate from the cladding tube, leading to a gap opening between these structural components initially in contact. Subsequent vibrations of the fuel rod assembly under dynamic coolant flow induce fretting wear on the cladding tube surface and ultimately leads to failure due to leakage of the fuel rod [3], [6].

Research has been directed at understanding the involved phenomena and alleviating the root causes responsible for GTR fretting wear. Out-of-reactor experiments study the effect on the wear characteristics of coolant flow induced vibrations and pressure fluctuations [7]-[9], of spring and dimple geometries [10]-[12], and of pre-sized gaps between the spacer grid and the fuel rods [9], [13]. In these experiments, the gaps between the springs and dimples and the fuel rod are generally pre-set to account for the effect of irradiation-induced contact relaxation and cladding creep at the end of life (cf. [14]). Precise determination of the gap size evolution under in-reactor conditions is challenging because of the synergistic contributions from irradiation growth and creep-induced dimensional changes of the spacer grid and clad, and coolant flow- 
induced vibrations that cause wear. Modeling efforts have also generally focused on the effect of coolant flow-induced vibration on wear. Non-linear vibration models [15]-[17] have been used to study the effect of various flow parameters on the wear characteristics. Again, in these studies, a pre-set gap is generally assumed to account for the irradiation-induced gap opening, but the mechanisms contributing to the gap opening are not well understood. Moreover, frameworks that predict gap opening generally use phenomenological models for irradiation growth and creep (cf. Ref. [18]), assume no coupling between irradiation growth and creep, and have no consideration for the microstructure and/or material processing history.

The focus of the present work is to use physically-based models for irradiation growth and creep in a finite element framework to study the interactions between the spacer grid and the fuel rod under in-reactor conditions and predict the gap opening. Irradiation growth and creep are material - and microstructure - dependent phenomena and have been studied extensively in the past (cf. [5], [19]). We have recently developed a constitutive crystal plasticity framework for modeling irradiation growth and creep in hexagonal closed packed polycrystals, specifically $\mathrm{Zr}$ and its alloys [20]. This framework accounts for the creation of point defects during radiation cascades, their interaction with line dislocations and grain boundaries, nucleation and growth of dislocation loops, and their contribution to strain at the level of single crystals (grains). Intergranular interactions are solved using the visco-plastic self-consistent polycrystal plasticity (VPSC) model [21]. This crystal plasticity framework can account for the effects of texture and evolving microstructure on the deformation behavior and was used to predict the irradiation growth behavior of cold-worked Zircaloy-2. In the present work, we have interfaced this irradiation growth and creep framework with the finite element code ABAQUS in the form of a material model, to solve for texture-dependent deformation at the component level. This finite element framework is used to study interactions between the spacer grid and fuel rod, and the evolution of gap opening as a function of irradiation.

The predicted gap opening from these simulations may be used in vibration analysis experiments and calculations to study the ensuing wear behavior, and also guide the design of nuclear fuel rod assemblies.

\section{Methods}

\subsection{Crystallographic model for irradiation growth and creep}

The reaction-diffusion model is discussed in detail in Refs. [20], [22], [23] and only the main characteristics and assumptions are described in the following. The neutron flux induces radiation cascades which create vacancies, self-interstitials, and interstitial clusters. The model uses four crystallographic directions to describe strains and dislocation Burgers vectors: the three basal compact directions, $j \equiv \boldsymbol{a}_{1}, \boldsymbol{a}_{2}, \boldsymbol{a}_{3}$, and the c-axis direction, $j \equiv \boldsymbol{c}$. The average concentrations of vacancies and interstitials in the effective medium are denoted by $c_{v}$ and $c_{i}$, respectively, and they are assumed to diffuse isotropically. The model assumes that equal number of self-interstitial atom (SIA) clusters are generated along each basal direction, $j \equiv \boldsymbol{a}_{1}, \boldsymbol{a}_{2}, \boldsymbol{a}_{3}$, and keeps track of their individual concentrations, $c_{i c}^{j}$, separately. In addition, the SIA clusters diffuse uni-directionally along the basal directions, $j \equiv \boldsymbol{a}_{1}, \boldsymbol{a}_{2}, \boldsymbol{a}_{3}$. Dislocation lines are projected along $j \equiv \boldsymbol{a}_{1}, \boldsymbol{a}_{2}, \boldsymbol{a}_{3}, \boldsymbol{c}$ and dislocation loops form on the prism and basal planes with 
Burgers vectors, $\boldsymbol{j} \equiv \boldsymbol{a}_{1}, \boldsymbol{a}_{2}, \boldsymbol{a}_{3}$ and $\boldsymbol{c}$, respectively. Absorption of point defects and SIA clusters at dislocation sinks (with density, $\rho^{j}$ ) leads to the climb of line dislocations and both, interstitialtype and vacancy-type loops. Interstitial clusters have a Burgers vector in the basal plane, diffuse unidirectionally, and interact only with dislocations with the same Burgers vector, that is $b=a_{1}, a_{2}, a_{3}$.

Interstitial loops (with number density, $N_{i}^{j}$, and mean radius, $r_{i}^{j}$ ) and vacancy loops (with number density, $N_{v}^{j}$ and mean radius, $r_{v}^{j}$ ) nucleate and grow on the prism and basal planes, although the characteristics of the model favor net interstitial capture at loops on the prism planes, and net vacancy capture at loops on the basal planes. The resulting climb of these dislocation loops and line dislocations contributes to positive growth strains, $\varepsilon_{\text {growth }}^{a_{1}}, \varepsilon_{\text {growth }}^{a_{2}}, \varepsilon_{\text {growth }}^{a_{3}}$, along the prismatic directions and negative growth strain, $\varepsilon_{\text {growth }}^{c}$, along the basal direction. Further, the radiation induced-defects are also absorbed at grain boundaries (GBs) [20], [24].

Under a steady state assumption, the crystallographic strain rates along the prismatic and basal directions associated with the climb of dislocation loops and line dislocations are given by [20]:

$$
\begin{gathered}
\dot{\boldsymbol{\varepsilon}}_{\text {climb }}^{j}=G_{N R T}\left(1-f_{r}\right) f_{i c}\left(\frac{1}{3}-\frac{\rho^{j}}{\sum_{j} \rho^{j}+\sum_{j} \sum_{m} k_{m}^{j 2}}\right) ; j \equiv \boldsymbol{a}_{1}, \boldsymbol{a}_{2}, \boldsymbol{a}_{3} ; m \equiv \boldsymbol{x}^{\prime}, \boldsymbol{y}^{\prime}, \boldsymbol{z}^{\prime} \\
\dot{\boldsymbol{\varepsilon}}_{\text {climb }}^{j}=-G_{N R T}\left(1-f_{r}\right) f_{i c} \frac{\rho^{j}}{\sum_{j} \rho^{j}+\sum_{j} \sum_{m} k_{m}^{j 2}} ; j \equiv \boldsymbol{c} ; m \equiv \boldsymbol{x}^{\prime}, \boldsymbol{y}^{\prime}, \boldsymbol{z}^{\prime}
\end{gathered}
$$

where, $G_{N R T}$ is the Norgett-Robinson-Torrens (NRT) defect production rate [25] for vacancies and interstitials, $f_{r}$ is the fraction of point defects that recombine during the cascade, and $f_{i c}$ is the fraction of interstitials that form clusters. Here, $k_{m}^{j 2}$ is the sink strength of grain boundaries (GBs) for point defects (the superscript, $j$, indicates the Burgers vector direction, and the subscript, $m$, indicates the direction of the main axes of the ellipsoidal grain), defined as [20]

$$
k_{m}^{j 2}=(\hat{\boldsymbol{m}} \cdot \hat{\boldsymbol{j}}) \frac{1 / R_{m}^{2}}{\sum_{m} 1 / R_{m}^{2}} \frac{1}{R_{m}^{2}} \frac{3 \beta_{m}^{2}\left(\beta_{m} \operatorname{coth}\left(\beta_{m}\right)-1\right)}{\beta_{m}^{2}-3\left(\beta_{m} \operatorname{coth}\left(\beta_{m}\right)-1\right)} ; \beta_{m}=\sqrt{\sum_{j} \rho^{j}} R_{m} ; j \equiv \boldsymbol{a}_{1}, \boldsymbol{a}_{2}, \boldsymbol{a}_{3}, \boldsymbol{c} ; m \equiv \boldsymbol{x}, \boldsymbol{y}^{\prime}, \boldsymbol{z}^{\prime}
$$

where, $R_{m}$ is the length of the $m^{\text {th }}$ semi-axis of the ellipsoidal grain . Here, $\rho^{j}$ is the total density of dislocations with Burgers vector along crystallographic direction, $\boldsymbol{j}$, given as the sum of the line dislocation density, the areal (number of loops per unit volume times circumference) interstitial loop density, and the areal vacancy loop density, i.e.,

$$
\rho^{j}=\rho_{d}^{j}+2 \pi r_{i}^{j} N_{i}^{j}+2 \pi r_{v}^{j} N_{v}^{j}
$$


There is an additional contribution to growth due to the strain associated with defect absorption at grain boundaries and follows from the net flux of point defects at GBs. This has the form [20]:

$$
\dot{\varepsilon}_{G B}^{j}=-G_{N R T}\left(1-f_{r}\right) f_{i c} \frac{\sum_{m} k_{m}^{j 2}}{\sum_{j} \rho^{j}+\sum_{j} \sum_{m} k_{m}^{j 2}} ; j \equiv \boldsymbol{a}_{1}, \boldsymbol{a}_{2}, \boldsymbol{a}_{3}, \boldsymbol{c} ; m \equiv \boldsymbol{x}^{\prime}, \boldsymbol{y}^{\prime}, \boldsymbol{z}^{\prime}
$$

The total crystallographic strain rate due to irradiation growth is given as

$$
\dot{\varepsilon}_{\text {growth }}^{j}=\dot{\varepsilon}_{\text {climb }}^{j}+\dot{\varepsilon}_{G B}^{j} ; j \equiv \boldsymbol{a}_{1}, \boldsymbol{a}_{2}, \boldsymbol{a}_{3}, \boldsymbol{c}
$$

The 'macroscopic' strain rate tensor associated with irradiation growth, $\dot{\boldsymbol{\varepsilon}}^{\text {(growth })}$, is obtained by resolving the crystallographic strain rate, $\dot{\varepsilon}_{\text {growth }}^{j}$, along the main crystal axes, i.e.,

$$
\dot{\varepsilon}_{k l}^{(\text {growth })}=\sum_{j} \dot{\varepsilon}_{\text {growth }}^{j} b_{k}^{j} b_{l}^{j} ; j \equiv \boldsymbol{a}_{1}, \boldsymbol{a}_{2}, \boldsymbol{a}_{3}, \boldsymbol{c} ; k, l \equiv \boldsymbol{x}, \boldsymbol{y}, \boldsymbol{z}
$$

where, $b_{k}^{j}$ and $b_{l}^{j}$ are components of $\boldsymbol{b}^{j}$ along the $k$ and $\boldsymbol{l}$ directions of the crystal axes, respectively.

The presence of external stresses will lead to creep strain in the grains, in addition to irradiation growth. Moreover, incompatibility between grain-level growth strains and the surrounding medium strain will induce internal stresses, even in the absence of an external stress state. These grain-level internal stresses induce irradiation creep, which tends to relax the growth-induced stresses. It is therefore essential to account for irradiation creep in the polycrystal ensemble. We have implemented a crystallographic model for irradiation creep, inspired by the accepted empirical creep law used in steel deforming under irradiation creep and swelling (cf. [26]), but omitting the swelling term in this case. Such law assumes a linear dependence of creep rate with applied stress and radiation dose rate, $d \phi / d t$, of the form:

$$
\dot{\varepsilon}^{\text {creep }}=B_{0} \sigma \frac{d \phi}{d t}
$$

where, $\sigma$ is the effective stress, $B_{0}$ is the irradiation creep compliance, and $d \phi / d t$ is the radiation dose rate (in $\mathrm{dpa} \cdot \mathrm{s}^{-1}$ ). In our crystal plasticity framework we assume a similar phenomenological law to represent the shear rate associated with irradiation creep at the level of slip systems.

$$
\dot{\gamma}_{\text {creep }}^{j}=B \frac{\rho_{d}^{j}}{\rho_{\text {ref }}} \tau^{j} \frac{d \phi}{d t}
$$

where, $\tau^{j}$ is the resolved shear stress on slip system $j, B$ is the crystallographic irradiation creep compliance, and $\rho_{\text {ref }}$ is a reference line dislocation density. This expression is representative of a cascade unpinning-assisted dislocation glide mechanism for irradiation creep (cf. [27], [28]). The crystallographic shear rate is a function of the resolved shear stress $\tau^{j}$, and the density of line dislocations, $\rho_{d}^{j}$, that glide on slip system $j$. Only glissile line dislocations, $\rho_{d}^{j}$, but not the sessile dislocation loops, are activated by the resolved shear $\tau^{s}$ on the slip 
systems, and their contribution to deformation is proportional to the relative availability of dislocations. As discussed in Fidleris [5], irradiation-induced swelling is negligible in $\mathrm{Zr}$ alloys at the operation temperatures of interest and is therefore not assumed to be present in our constitutive model formulation.

\subsection{Polycrystal framework}

Here we use the anisotropic visco-plastic self-consistent (VPSC) polycrystal plasticity model developed by Lebensohn and Tomé [21] as a platform for implementing the growth and creep constitutive equations discussed above for individual grains, while accounting for texture, grain interaction with its surroundings, and grain shape. Within VPSC, the aggregate (polycrystal) is represented by a discrete set of individual crystal orientations (grains), to which a volume fraction is assigned in order to reproduce the measured texture. The response and properties of such aggregate are given by averaging the response and the properties of the individual constituent grains. VPSC keeps track of and evolves incrementally the stress, strain, crystal orientation, activity of each slip system in each grain, dislocation density on each slip system, etc.

VPSC treats each grain as an ellipsoidal visco-plastic inclusion embedded in an effective viscoplastic medium, both having fully anisotropic properties. The effective medium represents the 'average' environment 'seen' by each grain. When stress, strain rate, or irradiation are applied to the effective medium, an interaction between the medium and the grain (embedded inclusion) takes place and local stresses are induced in the inclusion by the effective medium. The stresses, which follow from solving the stress equilibrium equations, are uniform inside the inclusion, but deviate from the average stress in the medium depending on the relative properties and deformation trend of the medium and the inclusion.

The glide and climb of dislocations induces plastic shear and no resulting volumetric dilatation. Denoting $\boldsymbol{n}^{s}$ the normal to the slip plane, and $\boldsymbol{b}^{s}$ the direction of shear (Burgers vector) on slip system $s$, the resolved shear stress on the shear plane and along the shear direction associated with the stress tensor $\sigma_{i j}^{c}$ acting on the grain is given by

$$
\tau^{s}=b_{i}^{s} n_{j}^{s} \sigma_{i j}^{c}=m_{i j}^{s} \sigma_{i j}^{c}
$$

where, the Schmid tensor $m_{i j}^{s}=\frac{1}{2}\left(b_{i}^{s} n_{j}^{s}+b_{j}^{s} n_{i}^{s}\right)$ projects the stress on the shear plane along the shear direction. The strain rate tensor associated with shear creep rates, $\dot{\gamma}_{\text {creep }}^{\text {s }}$ (Eq. (9)) in system $s$ is

$$
\dot{\varepsilon}_{i j}^{(\text {creep })}=\sum_{s} m_{i j}^{s} \dot{\gamma}_{\text {creep }}^{s}
$$

Adding the growth and creep contributions (Eqs. (7) and (11)), the total strain rate in the grain is

$$
\dot{\varepsilon}_{i j}=\dot{\varepsilon}_{i j}^{(\text {growth })}+\dot{\varepsilon}_{i j}^{(\text {creep })}
$$

For representing the response of the effective medium (polycrystal), VPSC uses a linear tangent relation:

$$
\overline{\dot{\varepsilon}}_{i j}=\bar{M}_{i j k l} \bar{\sigma}_{k l}+\overline{\dot{\varepsilon}}_{i j}^{o}
$$


where, $\overline{\dot{\varepsilon}}_{i j}$ and $\bar{\sigma}_{k l}$ are the overall (macroscopic) magnitudes, and $\bar{M}_{i j k l}$ and $\overline{\dot{\varepsilon}}_{i j}^{o}$ are the macroscopic creep compliance and macroscopic growth rate, respectively. The growth of the polycrystal takes place in the absence of applied stress. But, as mentioned earlier, internal stresses in the grains are induced by growth. Solving the inclusion-medium interaction leads to an 'interaction equation', which relates the stress and strain rate in the inclusion with equivalent average quantities in the effective medium

$$
\left(\dot{\varepsilon}_{i j}-\overline{\dot{\varepsilon}}_{i j}\right)=-\tilde{M}_{i j k l}\left(\sigma_{k l}-\bar{\sigma}_{k l}\right)
$$

where,

$$
\tilde{M}_{i j k l}=(I-S)_{i j m n}^{-1} S_{m n p q} \bar{M}_{p q k l}
$$

and, $\boldsymbol{S}$ is the visco-plastic Eshelby tensor. Equation (14) indicates that the stress and the strain rate in the inclusion differ, in general, from the ones of the effective medium. The macroscopic moduli, $\bar{M}_{i j k l}$, and $\overline{\dot{\varepsilon}}_{i j}^{o}$, are unknown a priori and need to be adjusted self-consistently by enforcing the condition that the stress and strain rate averaged over all grains has to be equal to the stress and strain rate of the effective medium, i.e.,

$$
\overline{\dot{\varepsilon}}_{i j}=\left\langle\dot{\varepsilon}_{i j}^{c}\right\rangle ; \bar{\sigma}_{k l}=\left\langle\bar{\sigma}_{k l}^{c}\right\rangle
$$

The self-consistent polycrystal approach described above has been used in the past by Tomé et al. [29]-[32] to model irradiation growth and creep of hexagonal polycrystals. The difference with the current application is that in the aforementioned papers single crystal growth and creep were assumed to be described by constant parameters that did not evolve with irradiation.

\subsection{VPSC-FE interface}

In this work, the constitutive deformation behavior used in the FE calculation is provided by VPSC. Effectively, each Gauss point in the FE mesh represents a polycrystalline aggregate with associated texture. VPSC solves the local boundary conditions imposed by the interface between VPSC and FE (referred to as VPSC-FE interface from here on) and then passes the deformed (stress and strain) state of the Gauss point to the FE code, which solves for global equilibrium of deformation in the FE mesh.

VPSC has previously been interfaced with the FE code, ABAQUS [33], and implemented in the form of a User MATerial subroutine (UMAT) [34], [35], and also with the BISON-CASL fuel performance code in the form of a material model [36]. In this work, a similar algorithm has been used, albeit with a modified convergence criterion.

The algorithm for the VPSC-FE interface [34] is briefly explained in the following. Before moving forward, it should be noted that standalone VPSC (referred as VPSC-SA from here on) only solves for the viscoplastic strain corresponding to a stress state (or vice-versa) and does not model the elastic strain. The VPSC-FE interface described here accounts for the 'macroscopic' elastic deformation as well. Also note that VPSC solves for deformation in the local (material) coordinate system, which is then rotated to the global (component level) coordinate system for FE calculations. 
An additive decomposition of the strain increment, $\Delta \boldsymbol{\varepsilon}$, into the elastic, $\Delta \boldsymbol{\varepsilon}^{e}$, and viscoplastic, $\Delta \boldsymbol{\varepsilon}^{v p}$, parts is assumed in the VPSC-FE interface, i.e., $\Delta \boldsymbol{\varepsilon}=\Delta \boldsymbol{\varepsilon}^{e}+\Delta \boldsymbol{\varepsilon}^{v p}$. The stress increment, $\Delta \sigma$, corresponding to this strain increment may be used to estimate the elastic strain increment, i.e., $\Delta \boldsymbol{\varepsilon}^{e}=\boldsymbol{C}^{-1}: \Delta \boldsymbol{\sigma}$, where $\boldsymbol{C}$ is the self-consistent elastic stiffness of the polycrystalline aggregate calculated by VPSC at the beginning of each deformation increment. The historydependent viscoplastic strain increment, $\Delta \boldsymbol{\varepsilon}^{v p}$, on the other hand, is a function of the stress state, $\sigma$ (rather than the stress increment), and the internal state variables (ISVs) in the constitutive model.

The FE code calls the VPSC-FE interface with an estimate of the stretch increment, $\Delta \boldsymbol{\varepsilon}_{F E}$, the rigid spin increment, $\Delta \boldsymbol{R}_{F E}$, and the time step increment, $\Delta t$. The VPSC-FE interface then solves for $\sigma$ corresponding to this strain increment using an iterative Newton-Raphson scheme. The trial stress at time, $t+\Delta t$, is estimated based on the elastic strain increment from the previous time step, i.e.,

$$
\sigma_{t+\Delta t}=\sigma_{t}+\Delta \sigma=\sigma_{t}+C: \Delta \varepsilon^{e}=\sigma_{t}+C:\left(\Delta \varepsilon-\Delta \varepsilon^{v p}\right)
$$

where, the subscript refers to the respective time increment. VPSC is called with this stress state, $\sigma_{t+\Delta t}$, to obtain the corresponding viscoplastic strain rate, $\dot{\boldsymbol{\varepsilon}}^{v p}$. VPSC-FE interface then calculates the residual between $\Delta \boldsymbol{\varepsilon}$ and $\Delta \boldsymbol{\varepsilon}_{F E}$ as:

$$
\boldsymbol{X}(\Delta \boldsymbol{\sigma})=\Delta \boldsymbol{\varepsilon}-\Delta \boldsymbol{\varepsilon}_{F E}=\boldsymbol{C}^{-1}: \Delta \boldsymbol{\sigma}+\dot{\boldsymbol{\varepsilon}}^{v p} \Delta t-\Delta \boldsymbol{\varepsilon}_{F E}
$$

If the convergence criteria (described in the following) is not satisfied in iteration $k$, a trial stress increment for the next iteration, $k+1$, is calculated as

$$
(\Delta \sigma)_{k+1}=(\Delta \sigma)_{k}-J_{N R}^{-1}\left((\Delta \sigma)_{k}\right): X\left((\Delta \sigma)_{k}\right)
$$

where, the Jacobian, $\boldsymbol{J}_{N R}$, of the Newton-Raphson iteration is given as

$$
\boldsymbol{J}_{N R}(\Delta \boldsymbol{\sigma})=\frac{\partial \boldsymbol{X}(\Delta \boldsymbol{\sigma})}{\partial(\Delta \sigma)}=\boldsymbol{C}^{-1}+\boldsymbol{M} \Delta t
$$

where, $\boldsymbol{M}$ is the viscoplastic tangent moduli computed by VPSC as part of the self-consistent calculations.

A weighted convergence metric (cf. [37]) is employed here to achieve faster convergence. The scalar convergence metric is weighted according to the largest component of the strain increment, $\Delta \varepsilon_{F E}$, such that

$$
\chi=\sqrt{\sum_{i} \sum_{j}\left(\frac{\left|\Delta \varepsilon_{F E}^{i j}\right|}{\max \left(\left|\Delta \varepsilon_{F E}^{i j}\right|\right)} X^{i j}\right)^{2}}
$$

where, $i$ and $j$ denote the respective indices of the tensor quantities.

Note that VPSC computes all quantities in the local coordinate system (the one in which the texture of the material is expressed), while the FE code computes all quantities in the global 
coordinate system. The VPSC-FE interface, therefore, rotates all deformation quantities from the global to the local coordinate system via the rotation tensor, $\boldsymbol{R}=\Delta \boldsymbol{R}_{F E} \cdot \boldsymbol{R}_{t}$ before passing them from the FE code onto VPSC (and vice-versa). Here, $\boldsymbol{R}_{t}$ is the total rotation at the end of time $t$. Also note that the tangent stiffness matrix, $\boldsymbol{C}^{T}$, required by some FE codes for calculation of the deformation increment for the next time step, is simply the inverse of the Jacobian used in the Newton-Raphson calculations in Eq. (20), i.e, $\boldsymbol{C}^{T}=\boldsymbol{J}_{N R}^{-1}$. This is therefore passed on to the calling FE code by the VPSC-FE interface. The algorithm for interfacing VPSC with the FE code is summarized in Fig. 1. The interface has been validated via simulation of irradiation growth in the quarter geometry of a cladding tube. This is described in Appendix A.

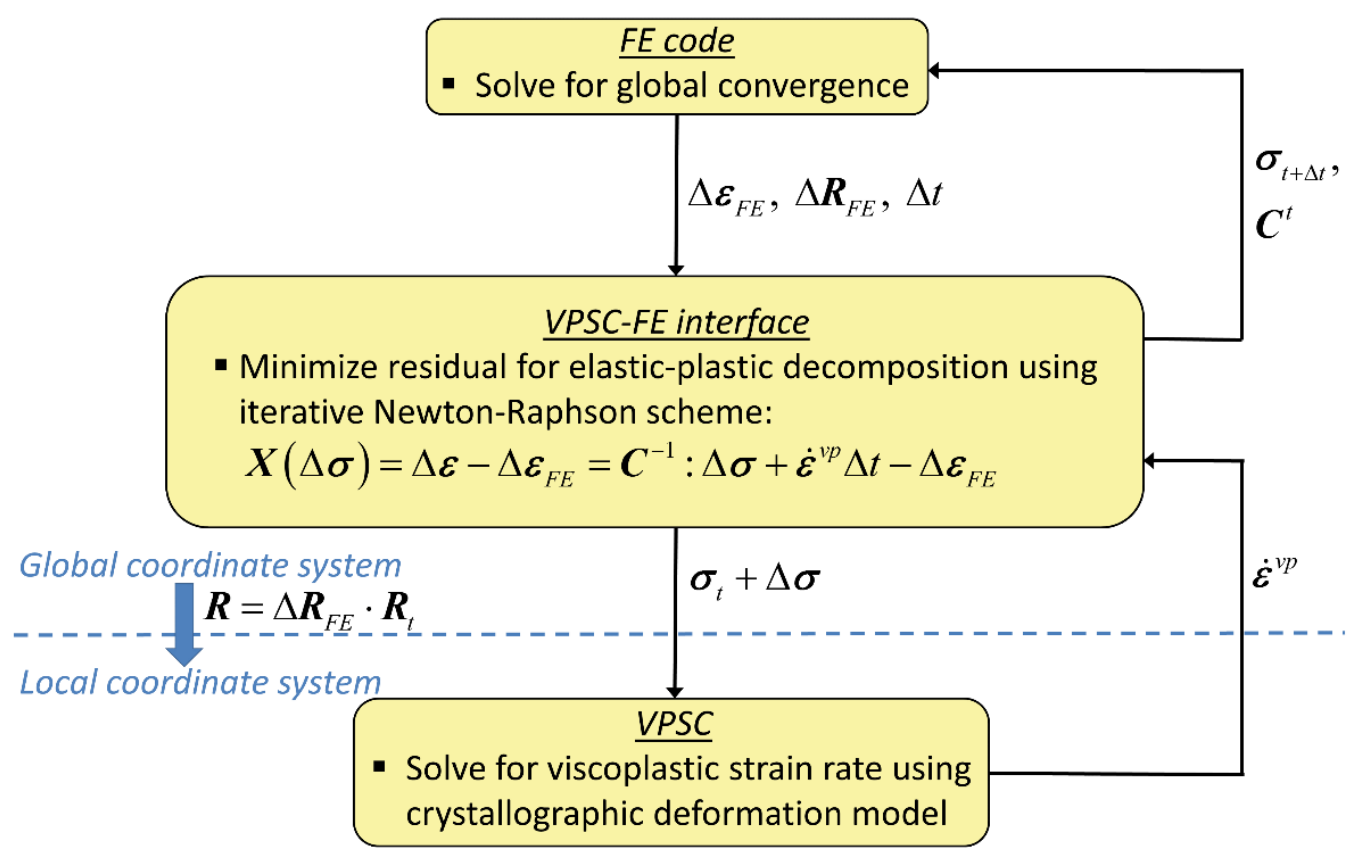

Figure 1. Algorithm for interfacing VPSC with the FE code.

\section{Simulation details}

\subsection{Simulation setup}

Figure 2(a) shows the geometry and mesh of the spacer grid and the cladding tube. Figures 2(b), 2(c), and 2(d) show the front and lateral views of one side of the spacer grid. Each side of the spacer grid has a length of $38.1 \mathrm{~mm}$, width of $12.776 \mathrm{~mm}$, and thickness of $0.229 \mathrm{~mm}$. The spring and dimple dimensions can be inferred approximately from Fig. 2. The cladding tube has an outer diameter of $9.5 \mathrm{~mm}$ and an inner diameter of $8.357 \mathrm{~mm}$, with a length of $38 \mathrm{~mm}$. The length of the fuel rod assembly (along the axial direction) represents the unit cell configuration of the springs and dimples on the spacer grid which repeats periodically every $38 \mathrm{~mm}$. Each side of the spacer grid is comprised of 6044 finite elements and the cladding tube is comprised of 23370 finite elements, so that there are a total of 47546 elements in the assembly. Finite elements with reduced integration (C3D8R) are used in ABAQUS. 
The bottom rim of the cladding tube is constrained in the axial $(z)$ direction but allowed to expand or contract radially. The bottom corner nodes of the spacer grid are constrained in all degrees of freedom to prevent rigid body motion. The initial configuration of the cladding tube and the spacer grid is such that the former is held by the latter via hard contact conditions between the cladding tube and the springs and dimples on the spacer grid. In order to model these interactions, the nodes on the cladding tube mesh and the spacer grid mesh are overlapping initially at regions where the springs and dimples are in contact. These overclosures are resolved prior to irradiating the assembly using the SHRINK parameter in ABAQUS, which automatically displaces the spacer grid and induces initial contact stresses in the elements.

In this work we do not attempt to model the effect of coolant flow-induced vibrations and forces on the clad-spacer grid system. Rather, our goal is to understand the evolution of contact forces and gap opening during irradiation. Such evolution is due to irradiation creep and irradiation growth of clad and spacer grid driven by the axial and circumferential stresses induced by pressure.
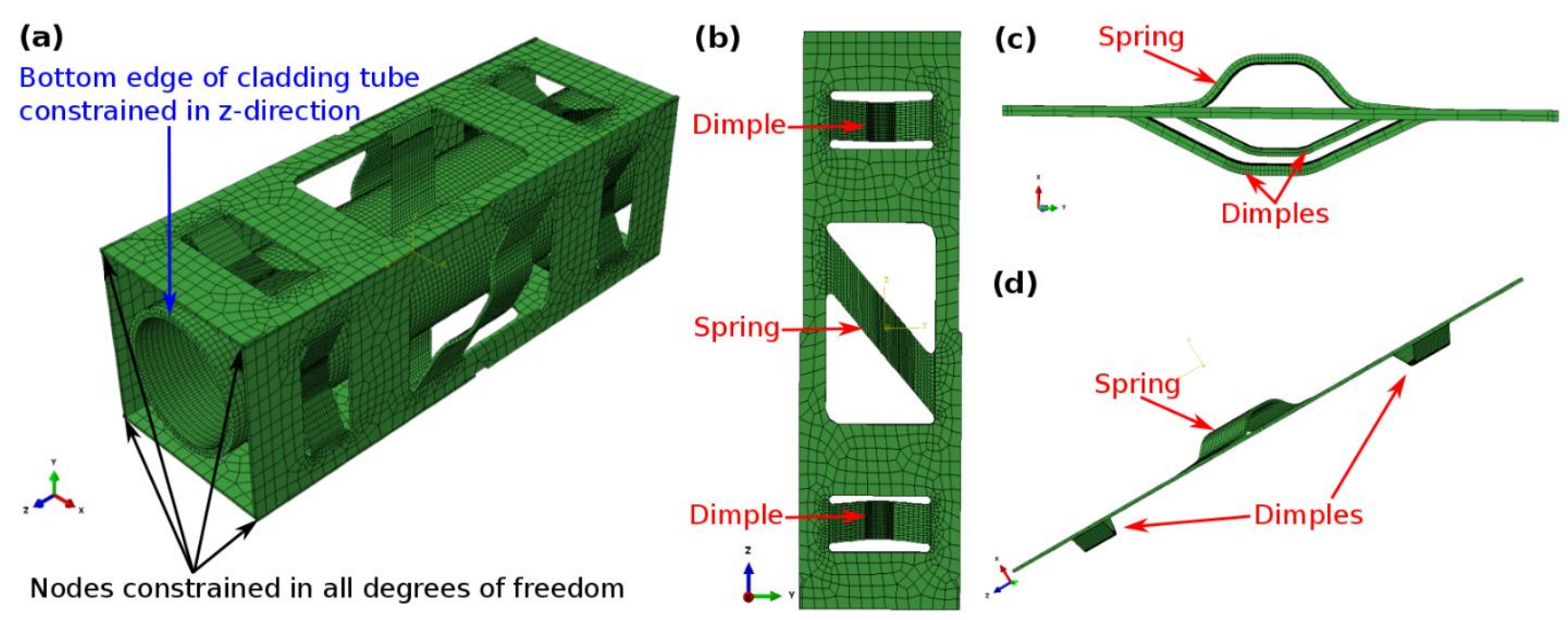

Figure 2. (a): Mesh and boundary conditions for the clad-spacer assembly, (b): front view of a side of the spacer grid, (c), (d): lateral views of a side of the spacer grid.

\subsection{Reduced texture for the cladding tube and spacer grid}

Finite element simulations are computationally expensive, especially when the material model calculations are performed at the level of grains using VPSC. A strategy to minimize the computational cost is to represent the texture using the minimum number of crystal orientations that will predict (within a $10 \%$ deviation margin) the same mechanical response of the aggregate as would be obtained if using the whole set of orientations. Concerning the cladding tube, we have adjusted our growth model [20] to dislocation density evolution reported by Holt et al. [38] for an irradiated Zircaloy-2 tube of which we do not have the discrete texture (their Fig. 6(c): basal pole figure of Chase Tube 002). As a consequence, we have used here an available tube texture of a cold-worked Zircaloy-2 cladding tube comprising 1144 orientations which has similar Kearn factors as those reported by Holt et al. [38]. From this texture, a reduced texture of only 7 orientations was derived. As for the spacer grid, a reduced texture comprising 13 
orientations was derived from an initial texture of 2428 orientations (the latter texture was measured using diffraction scans from the cross-section of the spacer grid springs at Oak Ridge National Laboratory, Oak Ridge, USA). Figure 3 shows the basal pole figures for the original and the corresponding reduced textures for the cladding tube and spacer grid used in our simulations. In reducing these textures, we enforced the Kearn factors of the reduced texture to be the same as those of the original texture. Kearn factors are defined as the second order moments of the basal poles, i.e.,

$$
F_{i}=\sum_{g} w^{g}\left(r_{i}^{g}\right)^{2}
$$

where, $w^{g}$ is the weight, and $r_{i}^{g}$ is the direction cosine of the c-axis of the $i^{t h}$ component of the $g^{\text {th }}$ orientation. Reduction of texture and resulting irradiation creep response of the polycrystal has been discussed in detail in Ref. [39]. Kearn factors of the cladding texture in Fig. 3(a) are: $F_{\text {axial }}=0.0864, F_{\text {hoop }}=0.5232, F_{\text {radial }}=0.3903$, and those of the reduced texture in Fig. 3(b) are identical. These factors are close enough to the ones reported by Holt et al. [38], which are 0.06, 0.49 and 0.45 , respectively. Kearn factors of the spring texture in Fig. 3(c) are: $F_{\text {rolling }}=0.1907$, $F_{\text {transverse }}=0.1102, F_{\text {normal }}=0.6991$, while that of the reduced texture in Fig. 3(d) are: $F_{\text {rolling }}=$ $0.1851, F_{\text {transverse }}=0.1051, F_{\text {normal }}=0.7098$. As demonstrated in Appendix A, irradiation growth and creep calculations performed using the reduced cladding texture compare well with experimental trends for cold-worked Zircaloy-2.

Note that the texture shown in Fig. 3(b) is the same for every element in the cladding tube when expressed along the radial, hoop, and axial directions of the tube. However, because the simulation geometry and FE computations are expressed in the laboratory Cartesian coordinates, the texture is rotated to laboratory axes for each FE comprising the cladding tube. Similarly, the texture shown in Fig. 3(d) is along the normal, transverse, and rolling directions of the rolled plate used to form the spacer grid, and is appropriately rotated to the laboratory axes for the FEs comprising each of the four sides of the spacer grid.
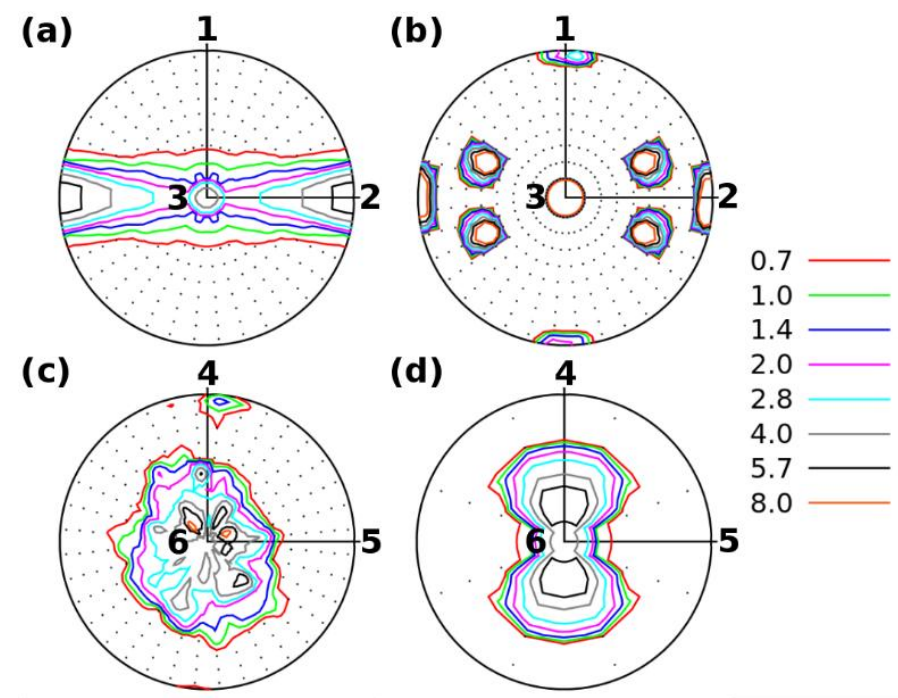

Figure 3. Basal pole figures for (a): cladding tube texture with 1144 orientations, (b): reduced cladding tube texture with 7 orientations, (c): spring texture with 2428 orientations, and (d): 
reduced spring texture with 13 orientations. Directions 1, 2, and 3 correspond to the axial, hoop, and radial directions of the cladding tube. Directions 4, 5, and 6 correspond to the rolling, transverse, and normal directions of the spring on the spacer grid sheet.

\section{Results and discussion}

\subsection{Distribution of pressure in springs and dimples}

Figure 4 shows the distribution of pressure in the springs and dimples on the spacer grid for various radiation doses. The maximum and minimum pressures for each of these contours is also specified. Sides 1, 2, 3, 4 and 1 are contiguous to each other. Further, the elements of the spacer grid in contact with the cladding tube are: spring 1 on side 1 , spring 2 on side 2, dimples 1 and 2 on side 3 (bottom to top), and dimples 3 and 4 on side 4 (top to bottom). This is schematically described in Fig. 5 to aid the interpretation of simulation results.

The cladding tube-spacer grid assembly was uniformly irradiated at a rate of $10^{-7} \mathrm{dpa} \cdot \mathrm{s}^{-1}$. A pressure of 15.5 $\mathrm{MPa}$ was applied on the outer surface (representative of the coolant pressure), and a pressure of $10 \mathrm{MPa}$ was applied on the inner surface of the cladding tube. The latter value is a first order approximation of the plenum pressure experienced by the fuel rod over the entire duration of the burnup in the range 6-15 MPa (cf. [40]-[42]). While no further external load was applied on the spacer grid, the pressure differential induces both, hoop and axial stresses. Further, the material model used for cold-worked Zircaloy- 2 was calibrated to the experimental irradiation growth data at $550 \mathrm{~K}$ from Ref. [38] (cf. [20]). Note that we have neglected the effects of thermal creep on the deformation behavior of Zircaloy-2 as it contributes second order effects in comparison to irradiation growth and creep at these relatively low homologous temperatures. Moreover, we have not modeled the fuel pellets in our FE simulations, which may contribute indirectly to the cladding-spacer grid interactions by impinging on the inner surface of the cladding tube. At the specified dose rate, a radiation dose of $\approx 3.15 \mathrm{dpa}$ is accumulated over one full power year. This implies that $10 \mathrm{dpa}$ corresponds to $\approx 3.2$ power years and $20 \mathrm{dpa}$ corresponds to $\approx 6.4$ power years.

The stresses on the spacer grid shown in Fig. 4 are induced primarily due to contact between the cladding tube and the springs and dimples. The stress concentration induced at the corners of the springs and dimples that are not in contact with the cladding tube are second order effects and are not expected to influence the gap opening between the spacer grid and the cladding tube significantly. 
(a) 0 dpa

Side 1/Spring 1
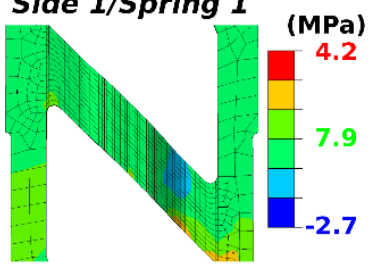

(b) 10.29 dpa

Side 1/Spring 1
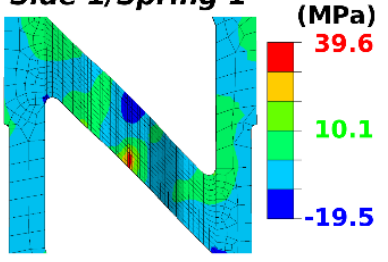

(c) 20 dpa
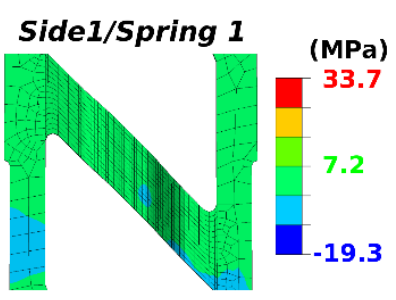

Side 2/Spring 2

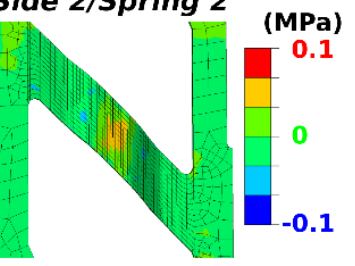

Side 3/Dimple 2

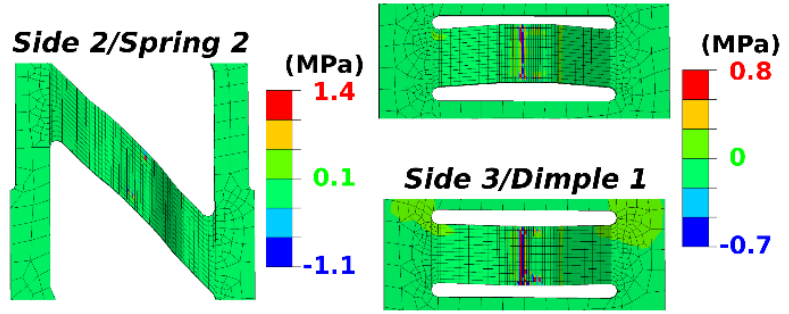

Side 3/Dimple 2

Side 2/Spring 2

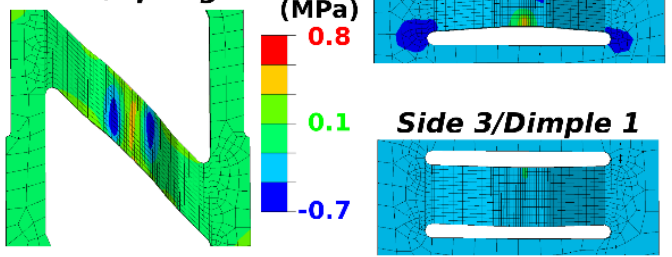

Side 3/Dimple 2

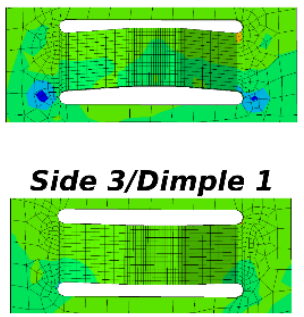

Side 4/Dimple 3

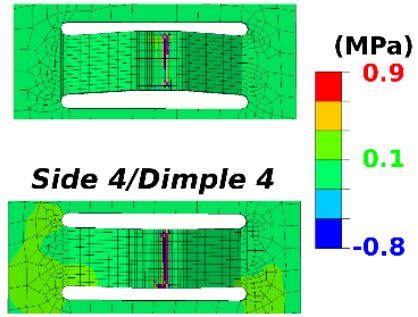

Side 4/Dimple 3

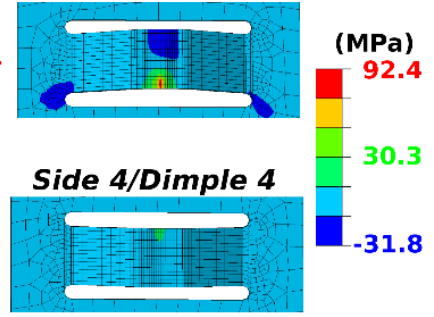

Side 4/Dimple 3
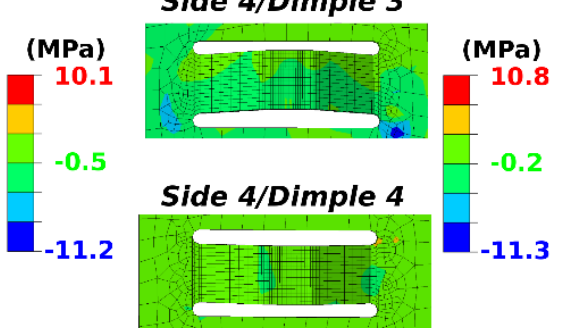

Figure 4. Distribution of pressure in the springs and dimples after (a): $0 \mathrm{dpa}$, (b): $10.29 \mathrm{dpa}$, and (c): 20 dpa dose. Note that the scales are different in each of these contours to highlight the stress concentration. 


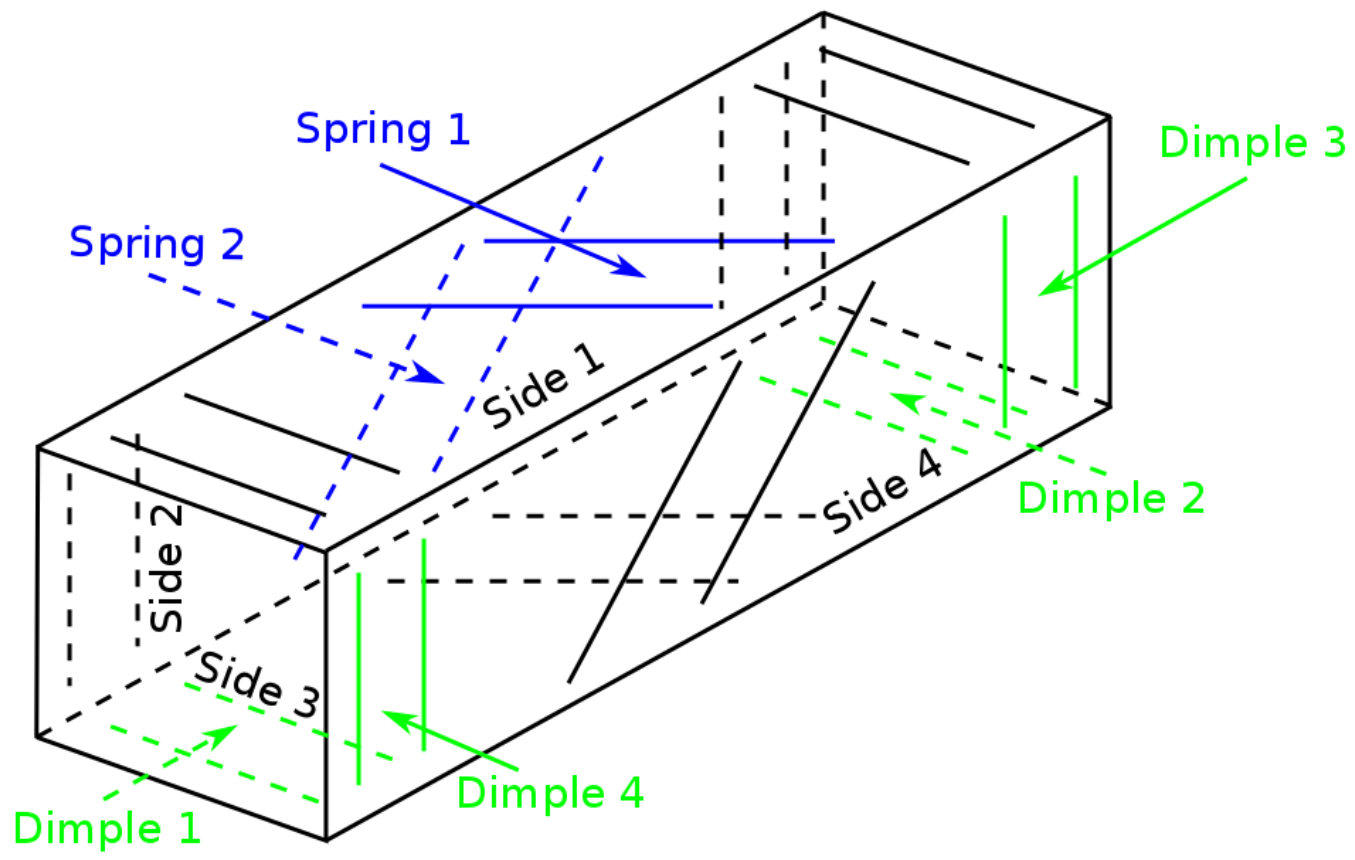

Figure 5. Schematic of the spacer grid showing the springs and dimples on the different sides of the spacer grid. Only the springs (in blue) and dimples (in green) on the inner sides of the spacer grid are labelled.

As seen in Fig. 4(a), there is a small pressure on the spring and dimple surfaces at 0 dpa dose. This is because the contact overclosures between the spacer grid mesh and the cladding tube mesh were resolved prior to irradiation. There is a thin line of contact on all the dimple surfaces, while no significant contact is observed on the spring surfaces to begin with. Figure 4(b) shows a considerable increase in pressure on the spring and dimple surfaces at $10.29 \mathrm{dpa}$ dose. The pressure goes up to $39.6 \mathrm{MPa}$ on the bottom edge of spring 1 on side 1 . It is still negligible on spring 2 on side 2 . A higher pressure is observed on the bottom edges of dimple 2 on side $3(\approx 80$ $\mathrm{MPa}$ ) and dimple 3 on side $4(\approx 92.4 \mathrm{MPa})$. The top edges of dimple 1 on side 3 and dimple 4 on side 4 exhibit lower pressures of the order $\approx 50 \mathrm{MPa}$. Figure 4(c) shows that the pressure is significantly relaxed in both the springs and dimples at $20 \mathrm{dpa}$ dose, as compared to $10.29 \mathrm{dpa}$. The pressure magnitudes are negligible, indicating no contact and a complete gap opening on these surfaces.

These observed trends can be correlated to the synergistic effects of various deformation phenomena (irradiation growth, contact pressure relaxation, grid and rod constraints) occurring in the spacer grid and the cladding tube. These will be discussed in detail in Section 4.3 using the analysis for gap opening distance between the springs and dimples and the cladding tube.

Our simulations also predict the nodal normal contact forces on the springs and dimples during irradiation. The simulated nodal contact forces lie in the range 0.01-1.7 N. Spring 1 on side 1 exhibits a maximum contact force of $0.84 \mathrm{~N}$ at 15.89 dpa dose, while spring 2 on side 2 exhibits a maximum contact force of $0.02 \mathrm{~N}$ at 0 dpa. Dimple 1 on side 3 exhibits a maximum contact force of $0.47 \mathrm{~N}$ at 5.49 dpa dose, while dimple 2 on side 3 exhibits a maximum contact force of $1.12 \mathrm{~N}$ at 14.29 dpa dose. Dimple 3 on side 4 exhibits a maximum contact force of $1.72 \mathrm{~N}$ at 
13.09 dpa dose, while dimple 4 on side 4 exhibits a maximum contact force of $0.29 \mathrm{~N}$ at $2.69 \mathrm{dpa}$ dose. While we have only performed a static analysis here (with no coolant flow), these contact forces are consistent with other studies in the literature. Jiang et al. [43] performed finite element simulations of the dynamic impact of the fuel rod with the spacer grid for a plastically deforming Zircaloy-4 cladding and spacer grid. They found the rod-to-spring contact force to be in the range $0.08-0.16 \mathrm{~N}$, and the rod-to-dimple contact force in the range 0.17-0.47 $\mathrm{N}$ [43]. Further, our model predictions are also consistent with the observation that the dimples generally experience higher contact force as compared to the springs [43].

\subsection{Evolution of contact opening}

Figure 6 shows the side view of the bottom edge of dimple 1 on side 3 of the spacer grid after 0 dpa and 20 dpa dose. This side view only highlights the change in nodal positions in the $\boldsymbol{x}$ - and $\boldsymbol{y}$ directions; there is an additional dimensional change due to irradiation growth along the $z$ direction, which is not highlighted here. It is seen that the dimple edge depresses more near the left end as compared to the right end on being irradiated from 0 dpa to $20 \mathrm{dpa}$ dose. This is possibly due to rotation of the cladding tube about the spring plane (to be discussed in detail in Section 4.3).

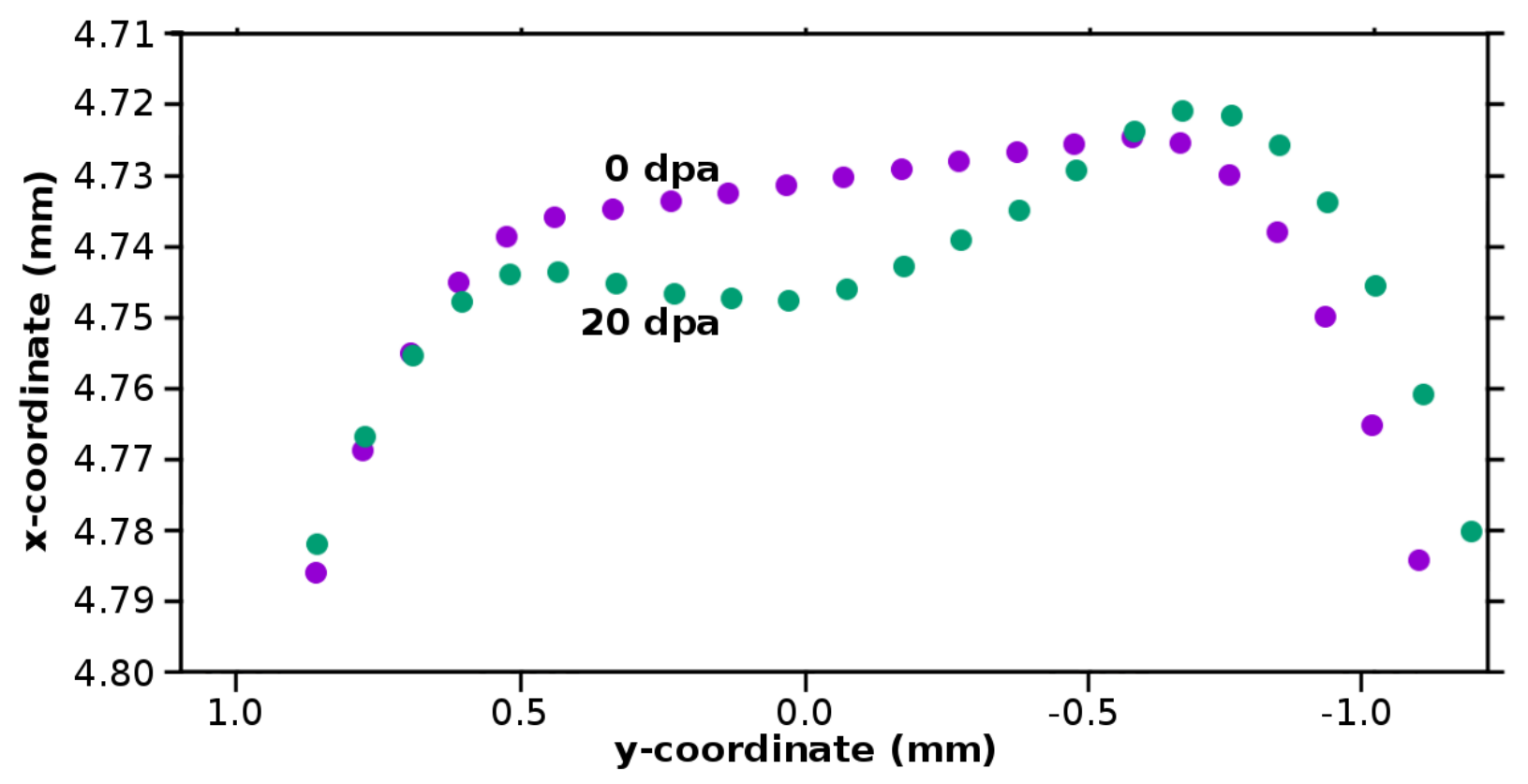

Figure 6. Side view of the bottom edge of dimple 1 on side 3 of the spacer grid after 0 dpa and 20 dpa dose.

However, as a result of the simultaneous deformation of the spacer grid and the cladding tube, it is difficult to infer the gap opening distance quantitatively over the entire contact surface. We use the contact opening distance variable, COPEN, in ABAQUS to get an average estimate of this. COPEN measures the distance between nearest nodes on the two contact surfaces (spring/dimple surface and cladding tube surface in the present case). Note that the surfaces in contact have to 
be specified a priori. At a given dose, we define gap as the arithmetic mean of COPEN over all nodes comprising the surface of a spring or a dimple, i.e.,

$$
\operatorname{Gap}_{\mathrm{dpa}}=\frac{1}{N_{\text {surface }}} \sum_{i=1}^{N_{\text {sufface }}} \operatorname{COPEN}_{\mathrm{dpa}}^{i}
$$

where, $\operatorname{COPEN}_{d p a}^{i}$ is the contact opening distance of the $i^{\text {th }}$ node at a given dpa dose, and $N_{\text {surface }}$ is the total number of nodes comprising the surface of the spring or dimple. Figure 7 shows the gap for the springs and dimples as a function of radiation dose. The initial non-zero values of gap measured at $0 \mathrm{dpa}$ dose are due the fact that all nodes of the spring/dimple surface defined as the 'contact surface' in the simulation are not in contact, and should be regarded as a reference value. As seen in the pressure contours of the dimple surfaces in Fig. 4(a), for example, only a thin strip on the dimple surface is actually in contact with the cladding tube. All other nodes on these dimple surfaces have non-zero COPEN values even at 0 dpa dose.

It is seen that there is no gap opening with radiation for spring 2 on side 2 , dimple 1 on side 3 and dimple 4 on side 4 . On the other hand, the gap opening keeps increasing starting at $\approx 5$ dpa dose, for spring 1 on side 1 , dimple 2 on side 3 and dimple 3 on side 4 . We define another measure to quantify the change in gap with radiation dose, $\Delta(\mathrm{Gap})_{\mathrm{dpa}}$, as

$$
\Delta(\mathrm{Gap})_{\mathrm{dpa}}=\mathrm{Gap}_{\mathrm{dpa}}-\mathrm{Gap}_{0 \mathrm{dpa}}
$$

The value of $\Delta(\text { Gap })_{20 \text { dpa }}$ is $15.1 \mu \mathrm{m}$ for spring $1,29.3 \mu \mathrm{m}$ for dimple 2 , and $30.3 \mu \mathrm{m}$ for dimple 3. This is the contribution of irradiation growth and creep to grid-to-rod gap opening under steady state conditions at $20 \mathrm{dpa}$ dose. Note that we have not considered the effect of fretting wear due to frictional contact between the spacer grid and the cladding, which is also influenced by the turbulent coolant flow and associated vibrations. It is expected that fretting wear combined with irradiation-induced deformation would further enhance the grid-to-rod gap under dynamic coolant flow conditions. Nevertheless, our simulations predict that irradiation growth and creep have a non-negligible contribution to the gap opening. While there is not much quantitative data available in the literature regarding gap opening during in-reactor conditions, out-of-reactor experiments on fuel rod assemblies generally use a transverse displacement amplitude in the range 5-30 $\mu \mathrm{m}$ for grid-to-rod fretting wear tests [7], [43]. In another analytical study [18], a grid-to-rod gap of $10 \mu \mathrm{m}$ was predicted at end of life for Zircaloy-4 spacer grid and clad, with consideration for irradiation growth and creep using phenomenological constitutive models. In the aforementioned study, the predicted spring force relaxation compared favorably with relevant experiments [18]. Our finite element simulations predict gap opening in the same range as well. 

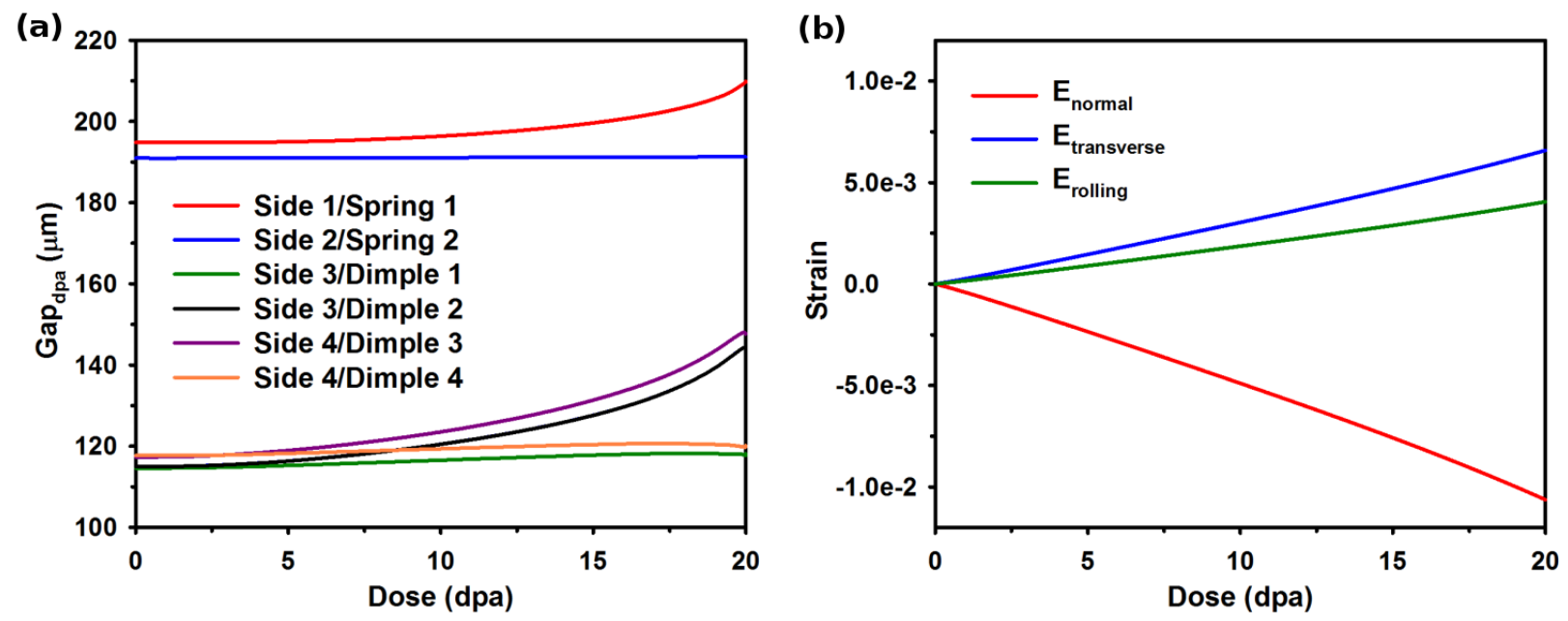

Figure 7. (a): Gap as a function of radiation dose for the springs and dimples, and (b): VPSC-SA predictions of growth strain for the spring texture in Fig. 3(d).

\subsection{Deformation mechanisms influencing gap opening}

Irradiation growth and creep are texture- and microstructure- dependent phenomena. Based on the texture given in Fig. 3(b), the cladding tube elongates along the axial direction and shrinks along the radial and hoop direction due to irradiation growth (cf. Fig. 13). As shown in Fig. 7(b), the spring texture in Fig. 3(d) results in the springs and dimples expanding along the rolling and transverse direction and contracting along the normal direction. This is schematically described in Figs. 8(a)-(c). Physically, the direction along the basal pole component of the texture exhibits contraction due to the aforementioned formation of vacancy loops along the $c$-axis, while the direction(s) perpendicular to this exhibits elongation due to the formation of interstitial loops along the prismatic directions, $\boldsymbol{a}_{1}, \boldsymbol{a}_{2}$, and $\boldsymbol{a}_{3}$. Eventually, the gap between the spacer grid and the cladding tube opens with accumulated dose as the contact forces relax due to irradiation growth and creep.

However, as a result of the mechanical configuration of the ensemble, the gap does not open symmetrically because the springs and dimples are not disposed symmetrically with respect to the axis of the tube. As shown in Fig. 5, the spacer sides with springs (sides 1 and 2) are adjacent to each other, while the spacer sides with dimples (sides 3 and 4) are adjacent to each other. As shown in Section 4.1, the contact forces are different in each of the springs and dimples. Visual inspection of the pressure contours in Fig. 4(b) suggests that spring 1 on side 1 is still in contact with the cladding tube at 10.29 dpa dose, while spring 2 on side 2 is no longer in contact. Also, dimple 2 on side 3 and dimple 3 on side 4, which are near the top end of the tube in this simulation show higher contact pressures as compared to dimple 1 on side 3 and dimple 4 on side 4 . This suggests that bending of the tube takes place about the bottom edge of dimple 2 and the top edge of dimple 1 on side 3 , and the bottom edge of spring 1 on (diametrically opposite) side 1. This is schematically described in Fig. 8(d). Moreover, since the spring is oriented at an angle with respect to the tube axis, the contact force exerted by the spring 1 on side 1 tends to rotate the cladding tube (Fig. 8(e)). Rotation of the tube in the plane of the spring has also been studied using an analytical model by Billerey [18]. These trends suggest that a multiaxial bending and rotation of the cladding tube takes place during irradiation. The tube is inclined 
against sides 1,4 , and 3, which are adjacent to each other, and out of contact with side 2 . With further irradiation up to $20 \mathrm{dpa}$ (cf. Fig. 4(c)), growth and creep relax the contact forces on the springs and dimples, such that they are completely out of contact with the cladding tube. These trends are also evident from the gap data presented in Fig. 7(a). It is seen that the gap opening does not change appreciably for spring 2 on side 2, while it increases with radiation dose for spring 1 on side 1 . Also, the dimples near the bottom end of the tube (dimple 1 on side 3 and dimple 4 on side 4) do not show appreciable change in the gap, while the gap increases with radiation dose for those near the top end (dimple 2 on side 3 and dimple 3 on side 4).

Note that we have not modeled fuel pellets in the present work. It is expected that expansion of the fuel pellets will arrest the 'inward' creep of the cladding tube and consequently lead to a nearly uniform gap size after a certain radiation dose. Also, we have not considered the effect of external loads on the springs and dimples due to contact with the adjacent fuel rods in this analysis. External loads on the outer surfaces of the springs and dimples are considered in Section 4.6.

(a)

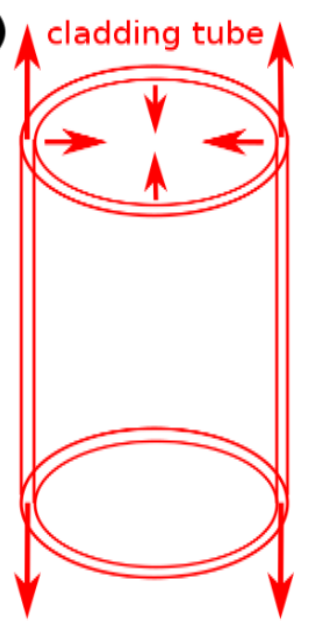

(b)

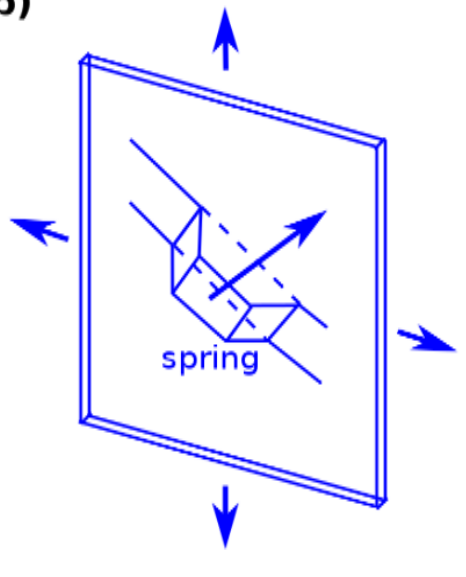

(c)

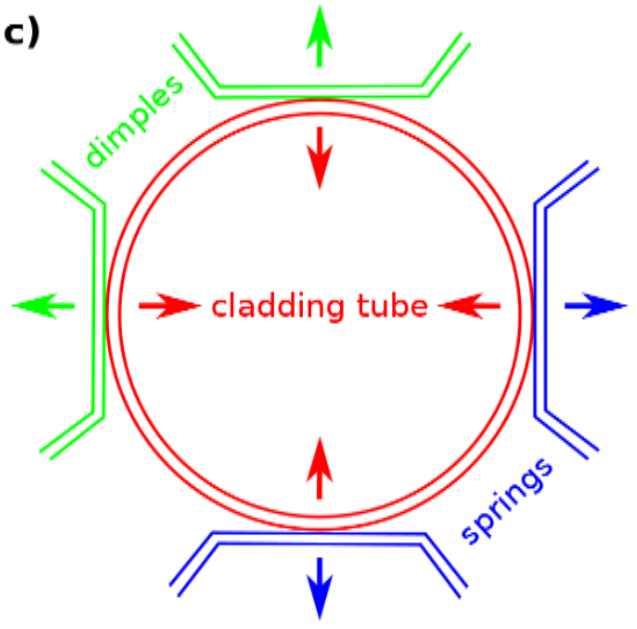

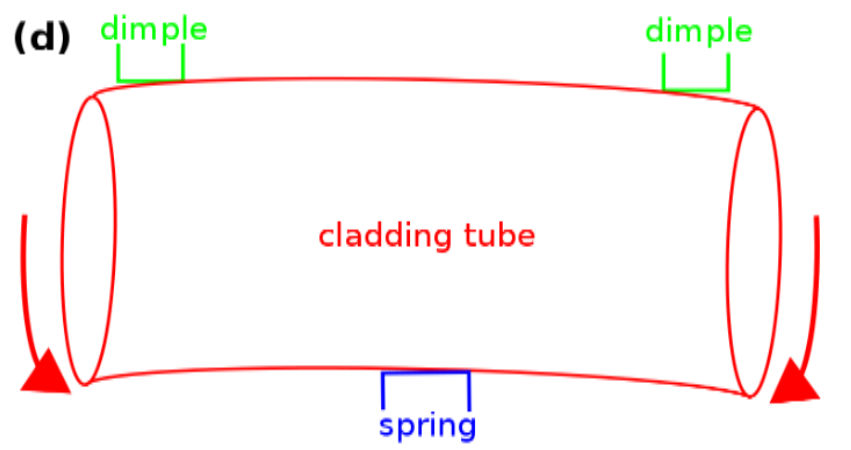

(e)

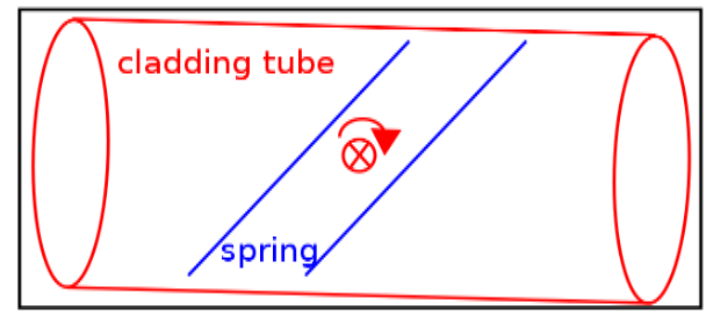

Figure 8. Schematic of texture-dependent deformation due to irradiation growth in (a): cladding tube, (b): spring on the spacer grid, (c): top view of the cladding tube and the springs and dimples on the spacer grid, (d): side view showing asymmetric bending of the cladding tube about one of the spring edges and the inner edges of the dimples, and (e): side view showing rotation of the cladding tube about the plane of the spring. Arrows indicate the direction of deformation. 


\subsection{Effect of texture}

In order to assess whether texture can be used to either reduce or eliminate the gap opening, we have performed a parametric study of the effect of texture on the gap opening between the spacer grid and the cladding tube. Results from three simulations are compared where the texture for the spacer grid was rotated by $90^{\circ}$ such that basal poles are either aligned with the normal (case T1), or the transverse (case T2), or the rolling direction (case T3) of the spring/dimple. Case 1 corresponds to the results presented in Section 4.1 through 4.3. Gap evolution and VPSC-SA predictions of growth strain for case $\mathrm{T} 1$ were presented in Fig. 7. The same results for case T2 and case T3 are presented in Figs. 9 and 10, respectively.

Comparing the simulation results in Fig. 7(a) with Fig. 9(a), it can be seen that the gap evolution profiles for case $\mathrm{T} 1$ and case $\mathrm{T} 2$ are nearly identical for all the springs and dimples. The gap at 20 dpa for spring 1 is slightly higher for case T2, as compared to case T1. The VPSC-SA calculations in Fig. 7(b) and Fig. 9(b) show that the strain along the normal and transverse directions are interchanged between case $\mathrm{T} 1$ and case $\mathrm{T} 2$ as a result of the rotation of the texture with respect to the spring geometry. The normal and transverse directions correspond either to the $\boldsymbol{x}$ - or $\boldsymbol{y}$-directions of the spacer grid plates (depending on their orientation), while the rolling direction corresponds to the $z$-direction in the laboratory coordinates. Based on these FE simulations, it is evident that expansion or contraction of the springs and dimples along either the rolling or transverse directions does not affect the gap opening significantly.

(a)

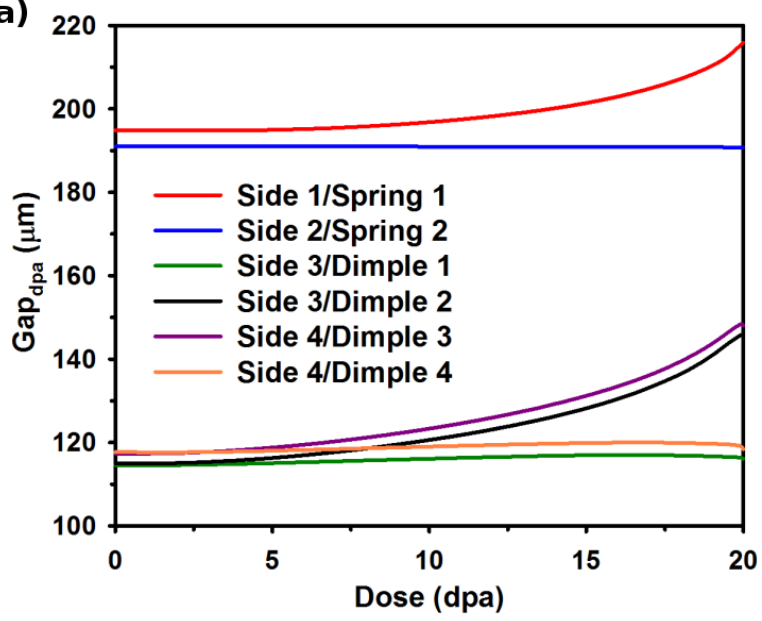

(b)

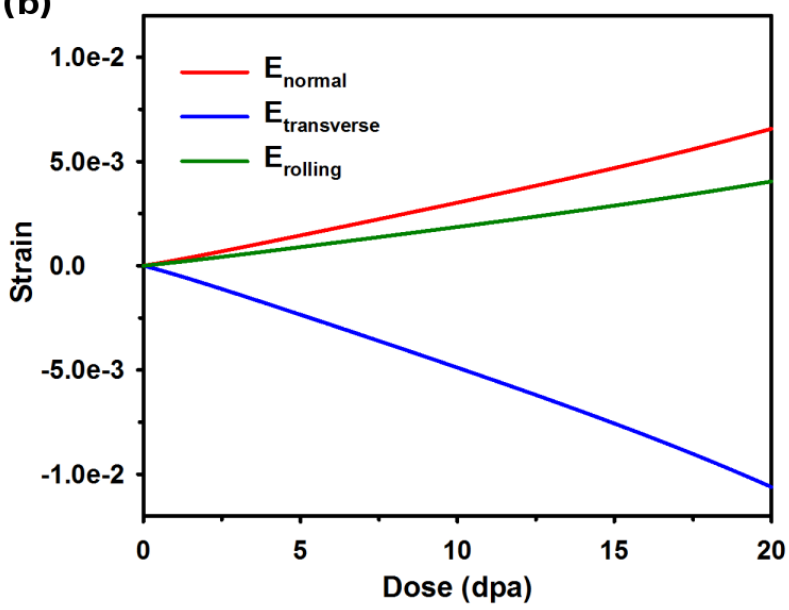

Figure 9. (a): Gap as a function of radiation dose for the springs and dimples, and (b): VPSC-SA predictions of growth strain for case $\mathrm{T} 2$, with basal poles along the transverse direction of the springs and dimples.

Figure 10(a) shows that case $\mathrm{T} 3$ has a different gap evolution profile as compared to cases $\mathrm{T} 1$ and T2. VPSC-SA predictions in Fig. 10(b) show that the spring texture, with basal poles along the rolling direction, results in contraction along the rolling direction and expansion along the normal and transverse directions. For case T3, the gap still does not increase appreciably for spring 2 on side 2, dimple 1 on side 3 and dimple 4 on side 4 . However, the gap is significantly 
larger for dimple 2 on side 3 and dimple 3 on side 4 . It is also marginally larger for spring 1 on side 1 as compared to case T1. Irradiation growth is a volume-conserving process and contraction of the spacer grid along the rolling direction results in expansion along the normal and transverse directions with radiation dose. Consequently, net expansion occurs along the lateral direction for the springs and dimples and this relaxes the contact forces with the cladding tube, resulting in larger contact opening.

At $\approx 18$ dpa dose, the gap starts to decrease again for dimple 2 on side 3 and dimple 3 on side 4 . Comparison with the contact pressure profiles (not presented here) shows that dimple 2 and dimple 3 are completely out of contact with the cladding tube at this point. Note that dimple 1 on side 3 , dimple 4 on side 4 , and spring 2 on side 2 were already out of contact before reaching this dose, implying that side 2 , side 3 , and side 4 are now completely out of contact with the cladding tube. The cladding tube starts rotating about its plane of contact with spring 1 on side 1 . Moreover, dimple 2 on side 3 and dimple 3 on side 4 are now completely relaxed (previously only the top edges of these dimples were in contact) and spatially relocated. As a result of the rotation of the cladding tube and the spatial relocation of nodes on dimple 2 and dimple 3 , we see a small drop in the gap for these past 18 dpa dose.

This parametric study highlights the effect of texture (physically representative of the cold-work history of the material) on gap opening. If the spring sheets are processed such that the basal poles are predominantly aligned with the rolling direction, it leads to a larger gap, which is detrimental from the fretting wear perspective (more gap will facilitate more vibrations during coolant flow and consequently more fretting wear). Dominant basal poles along the transverse direction do not change the gap significantly as compared to the spring sheet with basal poles along the normal direction. Simulations with different textures may be used to guide the design of spacer grids that have lower gap opening.

(a)

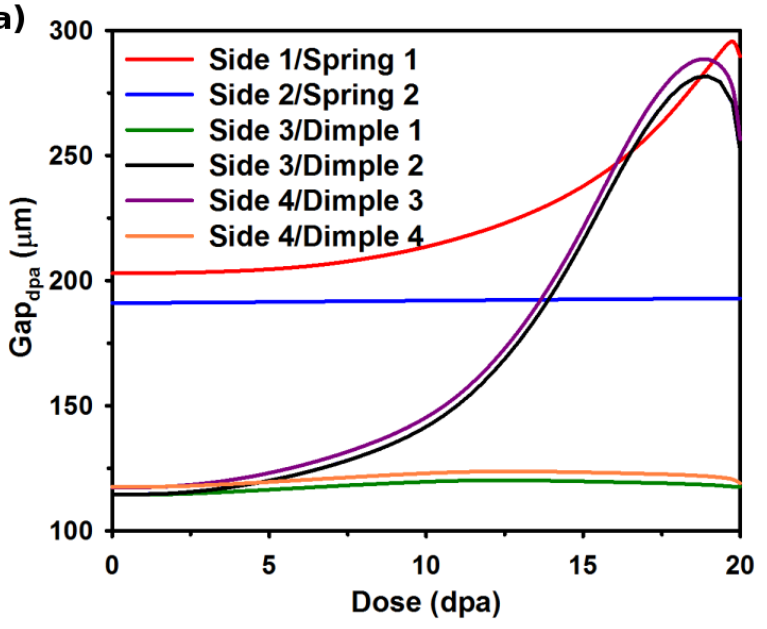

(b)

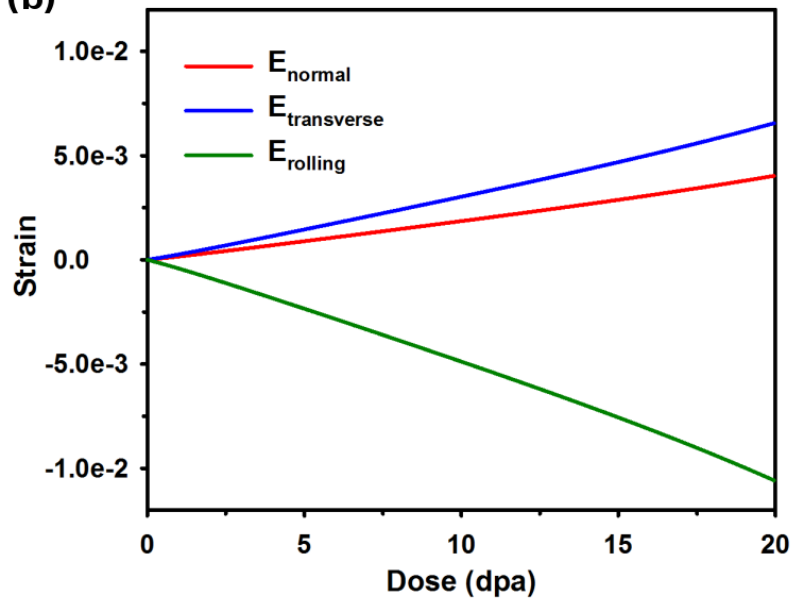

Figure 10. (a): Gap as a function of radiation dose for the springs and dimples, and (b): VPSCSA predictions of growth strain for case T3, with basal poles along the rolling direction of the springs and dimples.

\subsection{Effect of pressure reversal}


We performed a simulation where the pressures applied on the cladding tube were reversed from 15.5 $\mathrm{MPa}$ on the outer surface and $10 \mathrm{MPa}$ on the inner surface to $10 \mathrm{MPa}$ on the outer surface and 15.5 $\mathrm{MPa}$ on the inner surface. Effectively there is a net outward pressure of $5.5 \mathrm{MPa}$ acting on the cladding tube, which will induce creep along the hoop direction and so radial expansion of the cladding tube. All other boundary conditions, material parameters, and texture remain the same as in the simulation in Section 4.1.

Figure 11 shows the gap evolution profile for this case. It is seen that there is no appreciable change in gap evolution with radiation dose for either the springs or the dimples. This is because the radial displacement on the tube is pushing against the spacer grid, and irradiation growth and creep are not sufficient to relax the contact forces. While this loading scenario is hypothetical, it verifies that gap opening even under these steady state conditions is due to combined effects of pressure on the cladding tube and material-dependent irradiation growth and creep. In reactor conditions, rise in internal pressure may occur due to the release of gas bubbles from the fuel pellets and resulting expansion.

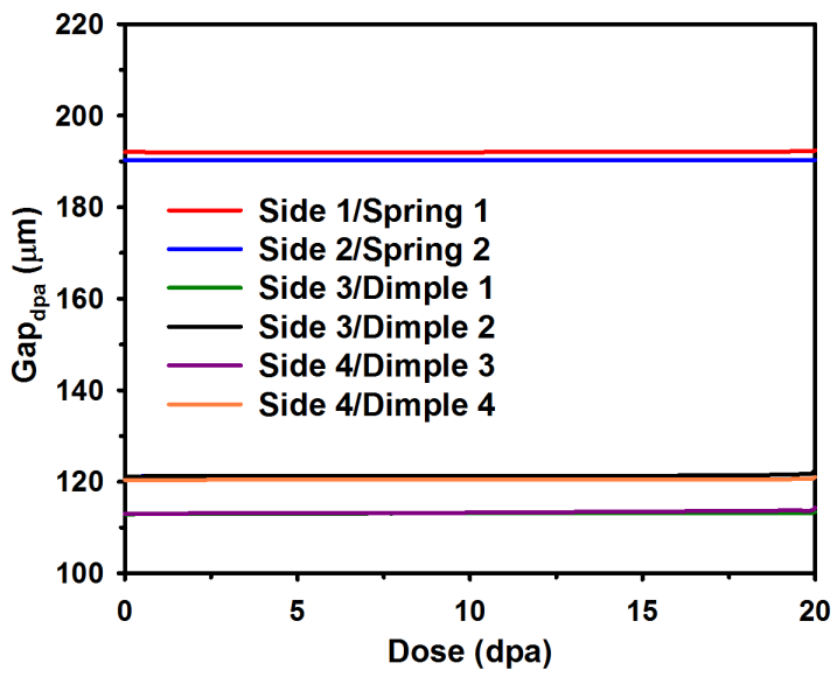

Figure 11. Effect of pressure reversal on the gap evolution profile for the springs and dimples.

\subsection{Effect of external loads on the spacer grid}

Until now we have only simulated gap opening in an isolated fuel rod inserted in a spacer grid that is not constrained laterally. However, a fuel rod assembly in a PWR consists of multiple fuel rods. For example, Yan et al. [6] discussed a Westinghouse fuel rod assembly with an array of 17 $\mathrm{x} 17$ fuel rods. They noted that the periphery rods in the fuel rod assembly at the fuel inlet region failed and started leaking, attributing this to insufficient rod support under high coolant flow rates [6]. In our simulations, we have applied distributed loads on the spring and dimple surfaces on the outer surface of the spacer grid in order to represent these fuel rod locations, albeit under steady state conditions, to a first order approximation. These loads are schematically shown in Fig. 12. Figure 12 shows the top view of the spacer grid, with different loads applied on springs and dimples on the outer surface of the spacer grid, and different constraints applied along the $\boldsymbol{x}$ and $\boldsymbol{y}$ - degrees of freedom at the corner nodes. In addition to these loads and constraints, the 
corner nodes on the bottom edge of the spacer grid are constrained in the axial $(z)$ direction for all cases. Red arrows in Fig. 12 indicate allowed displacements, depending on the presence or not of surrounding rods. Essentially, Case L1 represents a 'fully supported' fuel rod in the interior of the assembly, where it experiences loads on the springs and dimples on all four outer surfaces of the spacer grid due to contact with the neighboring fuel rods. No displacement along either $\boldsymbol{x}$ - or $\boldsymbol{y}$-directions on the corner nodes is assumed, to represent spatial constraints due to the neighboring fuel rods. Cases L2 and L3 represent periphery rods, where only one surface of the spacer grid is 'unsupported'. Either a dimple or spring on the outer surface of the spacer grid does not experience any external loads in these two cases. Cases L4, L5, and L6 represent fuel rods located at the outer corner of the assembly, where two surfaces of the spacer grid are 'unsupported'. A combination of springs and dimples on the outer surface of the spacer grid do not experience external loads in these cases. This comprises the full test matrix of cases where the outer surfaces of the spacer grid may not experience external loads due to fuel rods being located either at the periphery or outer corners.

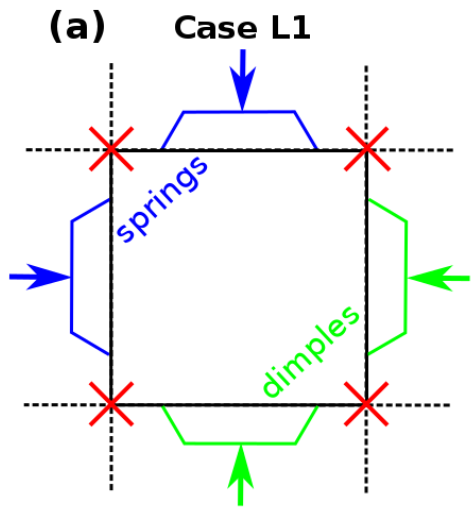

(d) Case L4

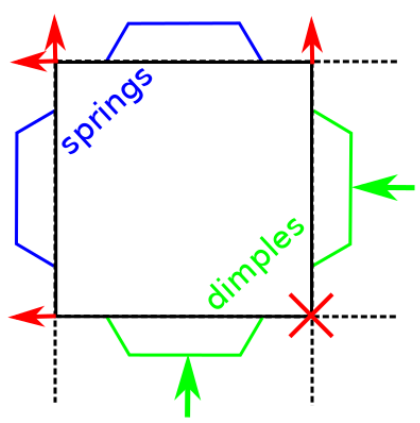

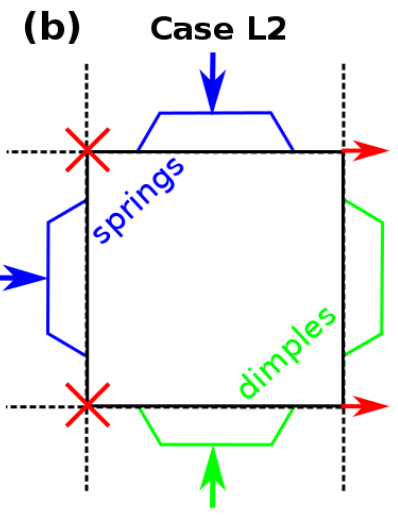

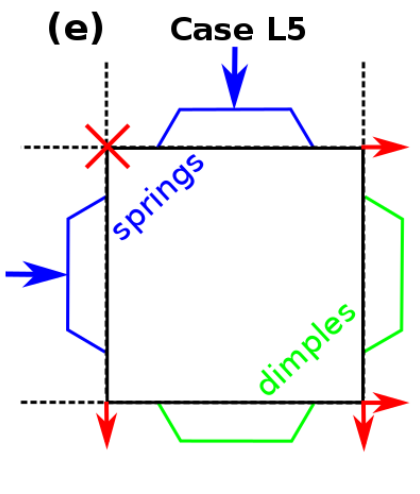

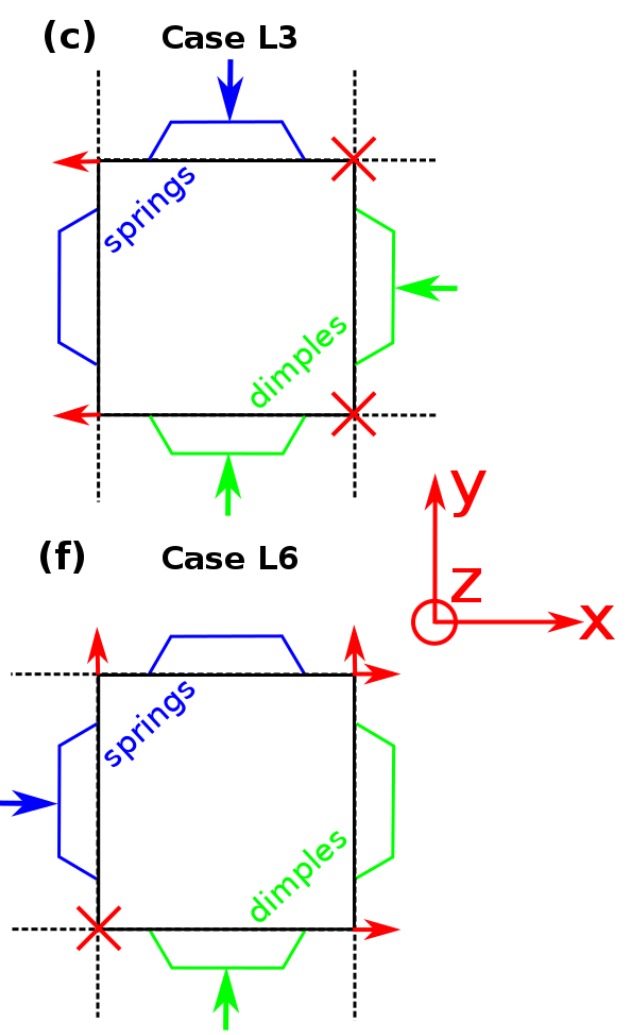

Figure 12. Schematic of external loads applied on the springs and dimples for various cases. Note that loads are applied on both dimples on the sides indicated by dimples. Corner nodes marked ' $\mathrm{X}$ ' in red are fixed in $\boldsymbol{x}$ - and $\boldsymbol{y}$ - degrees of freedom. Red arrows on the corner nodes indicate allowed displacement along the respective degree of freedom. Nodes on the bottom surface of the spacer grid are constrained axially ( $z$-degree of freedom), while nodes on the top surface are allowed to displace axially in all cases. Dotted black lines are shown to visually aid the reader regarding the presence of a neighboring fuel rod along a given surface of the spacer grid. 
A distributed load of $0.16 \mathrm{~N}$ was applied normal to each of the spring surface, and a distributed load of $0.47 \mathrm{~N}$ was applied normal to each of the dimple surface, where applicable. As discussed in Section 4.1, these are the maximum forces reported due to contact of the fuel rod with the springs and dimples from FE simulations, with material properties representative of plastically deforming Zircaloy-4 for the spacer grid and cladding tube [43]. Moreover, our simulations predict contact forces in the same range (cf. Section 4.1). All other simulation conditions remain the same as before.

Figure 13 shows the change in gap opening, $\Delta(\mathrm{Gap})_{\mathrm{dpa}}$, as a function of radiation dose for the springs and dimples for the different loading cases. Note that $\Delta(\mathrm{Gap})_{\mathrm{dpa}}$ is plotted here as opposed to $\mathrm{Gap}_{\mathrm{dpa}}$ previously, in order to highlight the minute differences in the gap opening profiles between the different cases. The change in gap at $20 \mathrm{dpa}, \Delta(\mathrm{Gap})_{20 \mathrm{dpa}}$, is also reported in Table 1 for the different cases. It is seen that, except for case L5, the gap decreases with radiation dose for spring 2 on side 2 for all cases. This indicates that the spring gets progressively more in contact or 'wraps around' the cladding tube with increasing radiation dose for these cases.

Cases L3, L4, and L6 are those in which external loads are not applied on the springs on at least one of the spacer sides. For these cases, the gap opening is generally higher as compared to cases L1, L2, and L5, especially on dimple 2 on side 3 and dimple 3 on side 4 (these dimples are adjacent to each other). As a result of enhanced gap opening, it may be expected that these rods will experience higher fretting wear. As mentioned earlier, case L3 is representative of a periphery fuel rod, with only one side of spacer grid unsupported externally, while cases L4 and L6 are representative of fuel rods on the outer corners of the assembly. Our simulation predictions provide a mechanistic understanding of why these fuel rods in the exterior may experience higher fretting wear and eventual failure. This also supports the hypothesis of higher fretting wear due to insufficient rod support, as discussed in Yan et al. [6]. Yan et al. [6] also noted that differences in coolant flow between fuel rods at the interior and at the periphery may lead to dominant vibration modes and contribute to eventual fretting wear. These are conditions that have not been modeled in the present work. 
(a)

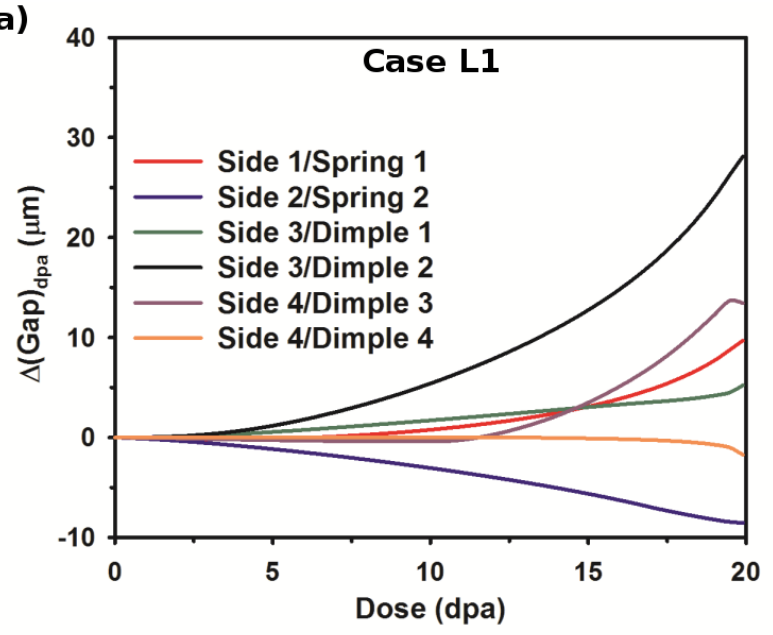

(c)

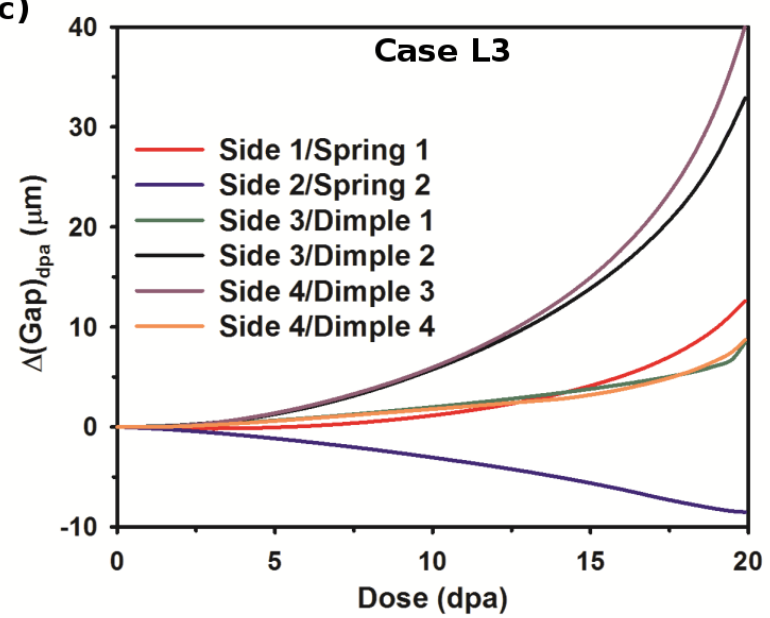

(e)

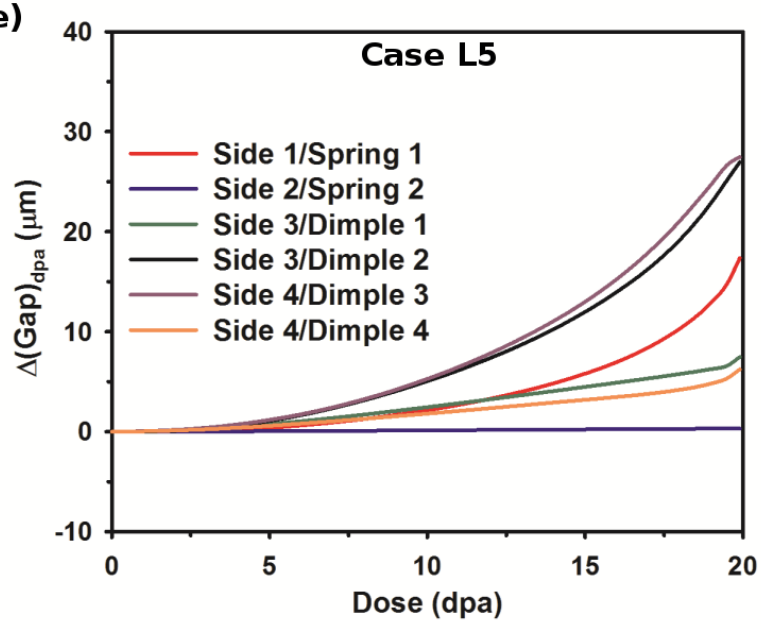

(b)

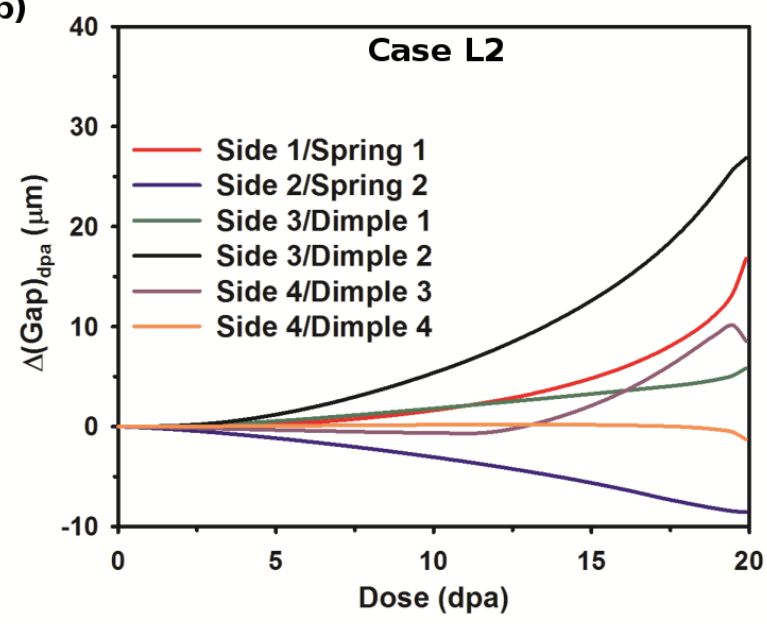

(d)

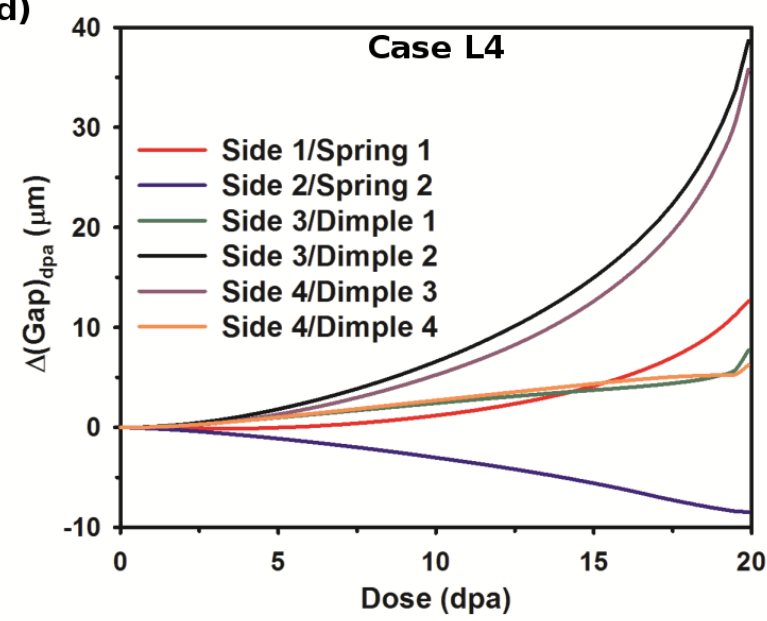

(f)

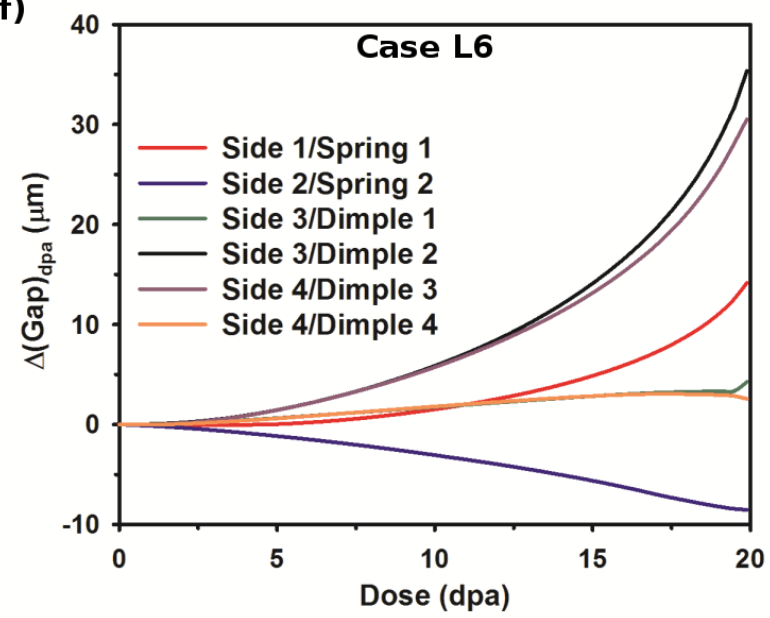

Figure 13. Gap as a function of radiation dose for the various external loads applied on the springs and dimples schematically described in Fig. 12. 
Table 1. Comparison of $\Delta(\mathrm{Gap})_{20 \mathrm{dpa}}$ for the various external loads applied on the springs and dimples schematically described in Fig. 12. All values are reported in $\mu \mathrm{m}$.

\begin{tabular}{|c|c|c|c|c|c|c|}
\hline Case \# & $\frac{\text { Side 1/ }}{\underline{\text { Spring } 1}}$ & $\frac{\text { Side 2/ }}{\text { Spring } 2}$ & $\frac{\text { Side 3/ }}{\text { Dimple } 1}$ & $\frac{\text { Side 3/ }}{\text { Dimple } 2}$ & $\frac{\text { Side 4/ }}{\text { Dimple } 3}$ & $\frac{\text { Side 4/ }}{\text { Dimple } 4}$ \\
\hline L1 & 9.70 & -8.54 & 5.21 & 28.11 & 13.44 & -1.73 \\
\hline L2 & 16.80 & -8.56 & 5.84 & 26.86 & 8.56 & -1.29 \\
\hline L3 & 12.58 & -8.52 & 8.44 & 32.87 & 40.04 & 8.71 \\
\hline L4 & 12.63 & -8.47 & 7.72 & 38.65 & 35.79 & 6.23 \\
\hline L5 & 17.36 & 0.32 & 7.47 & 26.97 & 27.49 & 6.24 \\
\hline L6 & 14.18 & -8.53 & 4.26 & 35.36 & 30.53 & 2.57 \\
\hline
\end{tabular}

\section{Conclusions}

A crystal plasticity framework for modeling irradiation growth and creep has been interfaced with the finite element code ABAQUS, to solve for microstructure- and texture-dependent deformation at the component level. This framework is used to study the interactions between the spacer grid and the cladding tube in a fuel rod assembly and the gap evolution between the springs and dimples (on the spacer grid) and the fuel rod as a function of irradiation.

Deformation mechanisms governing the gap opening have been identified and correlated to the texture-dependent material response. Contact pressures and contact forces on the springs and dimples have been measured from our simulations and are found to compare favorably with available data in the literature. A parametric study has been performed to highlight the effect of texture on the gap evolution profiles of the springs and dimples. It is found that the hypothetical presence of basal poles along the rolling direction of the spring sheet leads to significantly larger gap opening and is detrimental from the perspective of subsequent wear. We find that the current texture of the grid is preferable, since it tends to minimize the gap opening. Under the effect of reversed loading, with net outward pressure, the gap remains closed with irradiation, which would suggest that an initial pressurization of the cladding would improve the performance in what concerns lack of fretting. Finally, various combinations of external loads have been applied on the springs and dimples to simulate fuel rods in the interior, at the periphery and at the outer corners of the fuel rod assembly. It is found that loading conditions representative of fuel rods at the periphery and the outer corners show larger gap opening, for cases when there is no external force applied on the springs by neighboring fuel rods. Larger gap opening is expected to lead to higher fretting wear and eventual fuel rod leakage. This is in agreement with in-reactor data, where numerous cases of fuel rod failures were found to occur at the periphery of the fuel rod assembly [6]. This also agrees with the proposed hypothesis that insufficient rod support is one of the factors contributing to higher grid-to-rod-fretting wear [6].

\section{Acknowledgements}

This research is supported by the Consortium for Advanced Simulation of Light Water Reactors (CASL), an Energy Innovation Hub under US Department of Energy. Dr. Zupan Hu (University 
of Michigan) is thanked for providing the spacer grid mesh file. Dr. Donovan Leonard and Dr. Jun Qu (Oak Ridge National Laboratory) are thanked for providing the texture files for the spacer grid.

\section{Appendix A. Validation of the VPSC-FE interface}

In order to validate the VPSC-FE interface, irradiation growth and irradiation creep is simulated in the quarter geometry of a cylindrical cladding tube. The tube has an outer diameter of $9.5 \mathrm{~mm}$, inner diameter of $8.357 \mathrm{~mm}$, and a thickness of $0.5715 \mathrm{~mm}$. The length of the simulated tube is $38 \mathrm{~mm}$. The simulation geometry was meshed using 8-node reduced integration elements (C3D8R). This mesh is shown in Fig. 14 and has a total of 2400 elements, with 2 elements along the thickness. Axisymmetric boundary conditions were used such that two edges parallel to the axis of the tube are constrained in $\mathrm{x}$ - and $\mathrm{y}$-directions, respectively. One end of the tube is constrained along the axial (z-) direction, while the other end is left free to allow for axial growth. Further, an additional node along mid-circumference is constrained at the axial end of the tube in all degrees of freedom to prevent rigid body rotation. Different axial loads were applied on the end of the tube that is unconstrained to allow for axial growth. These boundary conditions are highlighted in Fig. 14.

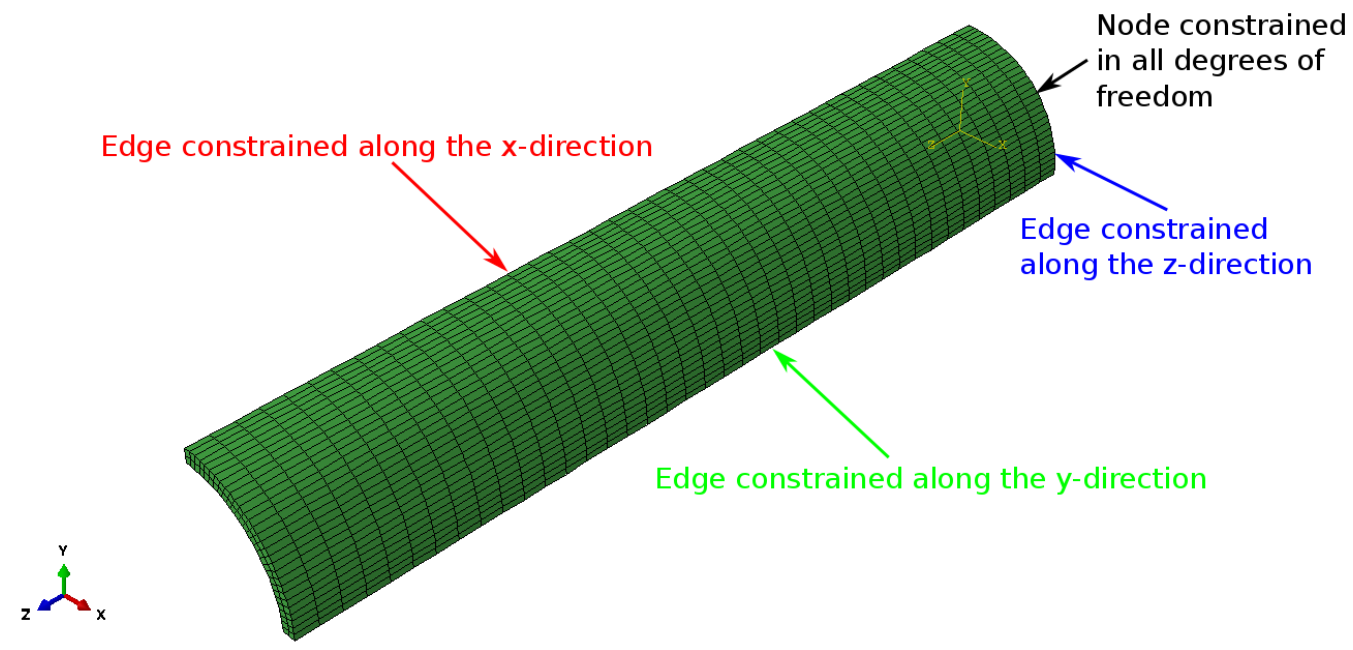

Figure 14. Mesh and boundary conditions for quarter geometry of the cladding tube.

A reduced cladding tube texture as in Fig. 3(b) was used for the FE simulations. Material parameters calibrated to the experimental growth data for Zircaloy-2 were taken from Ref. [20] and are given in Appendix B. The tube was irradiated at a dose rate of $10^{-7} \mathrm{dpa} \cdot \mathrm{s}^{-1}$ to $20 \mathrm{dpa}$ over 1000 time steps in the absence of any external loading.

Figure 15 shows the spatial distribution of strains in the tube after irradiation to 20 dpa dose. The directions 1,2, and 3 correspond to the radial, hoop, and axial directions of the cladding tube. The strain concentration near mid-circumference on the right end of the tube is because the midcircumference node on the right end of the tube is constrained in all degrees of freedom. But apart from that, a fairly homogeneous distribution of strain is predicted along the circumference 
and the length of the tube, as is expected form these irradiation conditions, the symmetry of the tube, and the absence of external loading. The tube exhibits negative growth strains along the radial and hoop directions and positive growth strain along the axial direction. This is a consequence of the texture of the tube which has been rolled along the axial direction and is in agreement with experimental evidence of irradiation growth in cold-worked Zircaloy-2 [38].
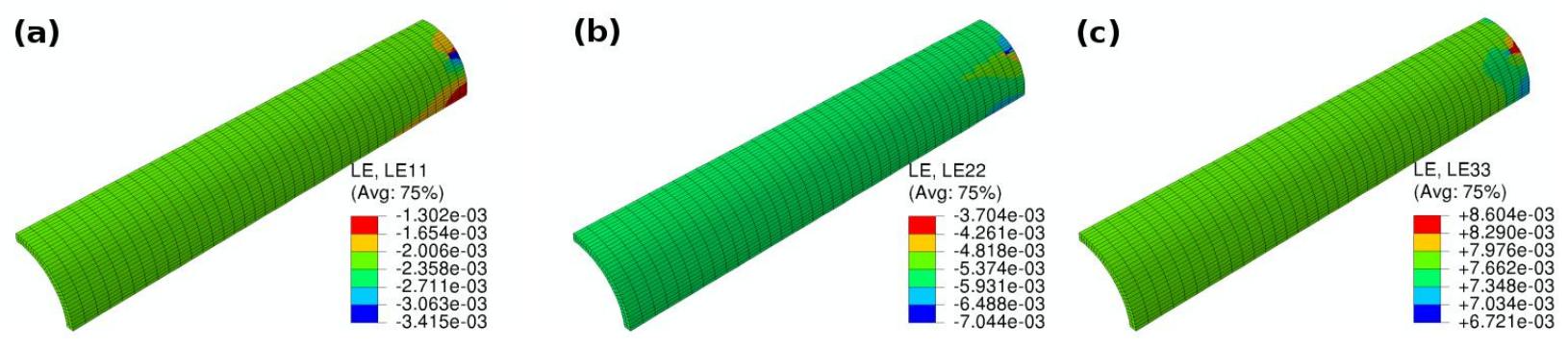

Figure 15. Distribution of (a): radial, (b): hoop, and (c): axial strains in the cladding after irradiation to 20 dpa.

For benchmarking purposes, we picked an element from the middle of the tube mesh and compared the temporal evolution of strains predicted by VPSC-FE (Finite Element) and VPSCSA (Stand Alone). Figure 16(a) shows the evolution of radial, hoop, and axial strains as a function of radiation dose for this element as compared to VPSC-SA predictions and experiments. No axial load was applied in these simulations. It is seen that predictions from the two simulations are in general agreement. The axial strain predictions from VPSC-SA and VPSC-FE calculations overlap and also compare well with experiments. Note that the material model parameters were calibrated to the experimental growth data using a representative cladding tube comprising of 1144 orientations given in Fig. 3(a) (cf. [20]). However, the reduced texture, with just 7 orientations and identical Kearn factors, is able to reproduce this growth behavior with reasonable precision and minor deviations between the VPSC-SA and VPSC-FE predictions for the radial and hoop strains with increasing dose. This may be due to the fact that VPSC-SA does not account for elastic deformation, which might be dominant at small strains of the order $10^{-3}$.

In order to verify the creep strain predictions, we perform a similar comparison between VPSCFE and VPSC-SA predictions with different applied axial loads. A reduced texture was used for the VPSC-FE simulations, while both full and reduced texture calculations were performed using VPSC-SA. These comparisons are shown in Fig. 16(b). It is seen that the VPSC-SA (both with full texture and reduced texture) and VPSC-FE predictions (with reduced texture) are identical for applied axial stresses of $100 \mathrm{MPa}$ and $200 \mathrm{MPa}$. Further, the 'macroscopic' irradiation creep compliance (slope) is calculated to be $3.03 \times 10^{-6} \mathrm{MPa}^{-1} \mathrm{dpa}^{-1}$ for the $100 \mathrm{MPa}$ case, and $3.06 \times 10^{-6} \mathrm{MPa}^{-1} \mathrm{dpa}^{-1}$ for the $200 \mathrm{MPa}$ case. The creep compliance values reported for Zircaloy-2 in the literature vary over a couple of magnitudes. Based on the data reported in Tinti [44], the irradiation creep compliance for cold-worked Zircaloy-2 was calculated to be in the range $2.5-7.5 \times 10^{-5} \mathrm{MPa}^{-1} \mathrm{dpa}^{-1}$ for stresses in the range $98-157 \mathrm{MPa}$ at $553 \mathrm{~K}$. In a separate work, Fidleris [5] estimated the irradiation creep compliance to be $1.53 \times 10^{-7} \mathrm{MPa}^{-1} \mathrm{dpa}^{-1}$ for 
Zircaloy-2 loaded to $181 \mathrm{MPa}$ at $553 \mathrm{~K}$. Our simulated irradiation creep compliance lies within this range. Calibration of the irradiation creep model parameters is discussed in Ref. [20].
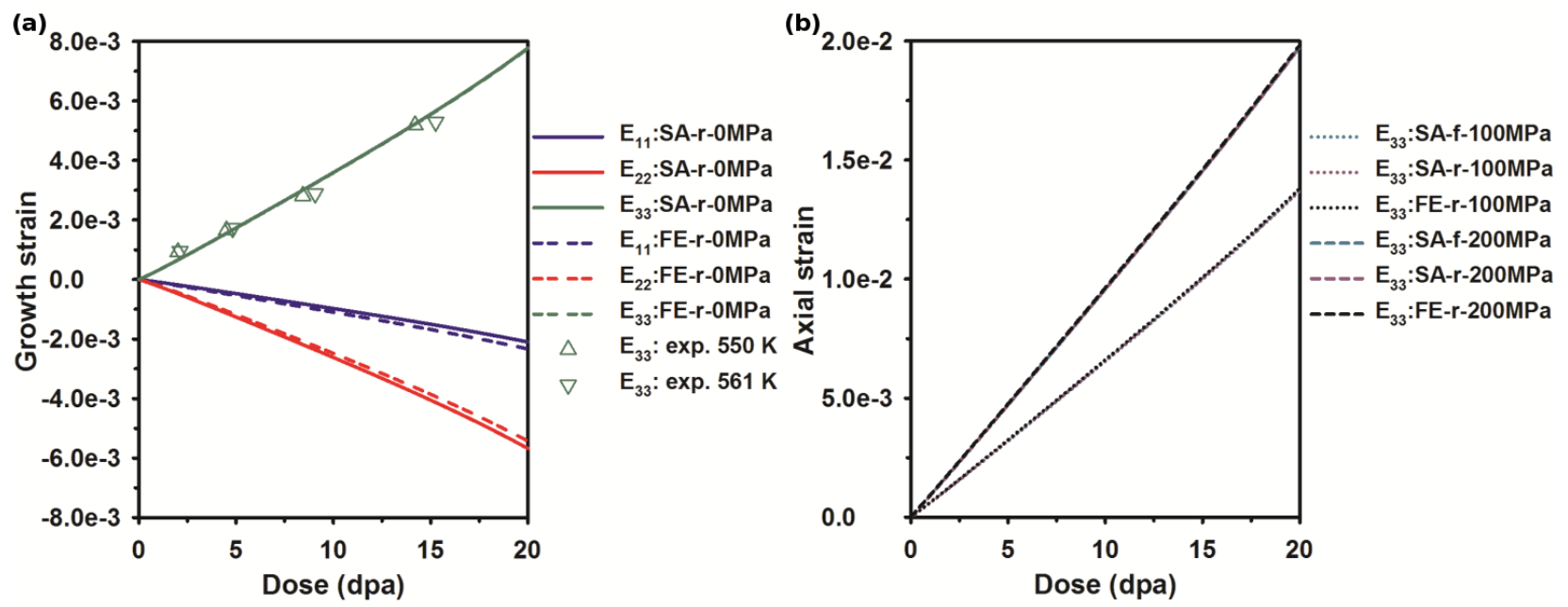

Figure 16. Evolution of (a) growth strains (in the absence of applied stress), and (b) axial strains as a function of radiation dose for an element on the circumference of the cladding tube mesh as compared to VPSC-SA predictions and experimental data [38]. Directions 1, 2, and 3 correspond to the radial, hoop, and axial directions of the cladding tube. SA refers to VPSC-SA predictions, while FE refers to VPSC-FE predictions. In the legend, $f$ refers to the full texture shown in Fig. 3(a), while $r$ refers to the reduced texture shown in Fig. 3(b). The stress values in the legend refer to the applied stress along the axial direction.

Appendix B: Model parameters for Zircaloy-2

\begin{tabular}{|c|c|c|}
\hline Parameter & Value & Meaning \\
\hline $\mathrm{C}_{11}, \mathrm{C}_{22}, \mathrm{C}_{33}$, & $143.5,143.5,164.9$ & Elastic constants in GPa (assumed to be the \\
\hline $\mathrm{C}_{12}, \quad \mathrm{C}_{13}, \mathrm{C}_{23}$ & $72.5,65.4,65.4$ & same as that for pure $\mathrm{Zr}$ ). Values taken from \\
\hline $\mathrm{C}_{44}, \quad \mathrm{C}_{55}, \mathrm{C}_{66}$ & $32.1,32.1,35.5$ & Refs. [45], [46]. \\
\hline$f_{r}$ & 0.97 & $\begin{array}{l}\text { Fraction of point defects that recombine during } \\
\text { the cascade. }\end{array}$ \\
\hline$f_{i c}$ & 0.13 & Fraction of interstitials that form clusters. \\
\hline$B$ & $5.0 \times 10^{-5} \mathrm{MPa} \cdot \mathrm{dpa}^{-1}$ & Crystallographic irradiation creep compliance. \\
\hline$\rho_{r e f}$ & $2.26 \times 10^{14} \mathrm{~m}^{-2}$ & $\begin{array}{l}\text { Weighting factor for line dislocation density in } \\
\text { irradiation creep model. }\end{array}$ \\
\hline$b^{j} ; j \equiv \boldsymbol{a}_{1}, \boldsymbol{a}_{2}, \boldsymbol{a}_{3}$ & $3.0 \times 10^{-10} \mathrm{~m}$ & $\begin{array}{l}\text { Magnitude of Burgers vector along the } \\
\text { prismatic directions. }\end{array}$ \\
\hline$b^{j} ; j \equiv c$ & $5.0 \times 10^{-10} \mathrm{~m}$ & $\begin{array}{l}\text { Magnitude of Burgers vector along the basal } \\
\text { direction. }\end{array}$ \\
\hline
\end{tabular}

\section{References}


[1] M. J. Pettigrew, "Flow-induced vibration of nuclear power station components," in 90th Annual Congress of the Engineering Institute of Canada, Halifax, Nova Scotia, 1976.

[2] C. A. Rusch, "Nuclear fuel performance: Trends, remedies and challenges," Journal of Nuclear Materials, vol. 383, no. 1-2, pp. 41-44, 2008.

[3] K.-T. Kim and J.-M. Suh, "Impact of nuclear fuel assembly design on grid-to-rod fretting wear," Journal of Nuclear Science and Technology, vol. 46, no. 2, pp. 149-157, 2009.

[4] K.-T. Kim, "A study on the grid-to-rod fretting wear-induced fuel failure observed in the 16×16KOFA fuel," Nuclear Engineering and Design, vol. 240, no. 4, pp. 756-762, 2010.

[5] V. Fidleris, "The irradiation creep and growth phenomena," Journal of Nuclear Materials, vol. 159, pp. 22-42, 1988.

[6] J. Yan, K. Yuan, E. Tatli, and Z. Karoutas, "A new method to predict Grid-To-Rod Fretting in a PWR fuel assembly inlet region," Nuclear Engineering and Design, vol. 241, no. 8, pp. 2974-2982, 2011.

[7] T. P. Joulin, F. M. Guérout, A. Lina, and D. Moinereau, "Effects of loading conditions and types of motion on PWR fuel rod cladding wear," pp. 1011-1018, 2002.

[8] M. H. Choi, H. S. Kang, K. H. Yoon, and K. N. Song, "Vibration analysis of a dummy fuel rod continuously supported by spacer grids," Nuclear Engineering and Design, vol. 232, no. 2, pp. 185-196, 2004.

[9] Y.-H. Lee and H.-K. Kim, "Fretting wear behavior of a nuclear fuel rod under a simulated primary coolant condition," Wear, vol. 301, no. 1-2, pp. 569-574, 2013.

[10] H.-K. Kim and Y.-H. Lee, "Influence of contact shape and supporting condition on tube fretting wear," Wear, vol. 255, no. 7-12, pp. 1183-1197, 2003.

[11] H.-K. Kim, Y.-H. Lee, and S.-P. Heo, "Mechanical and experimental investigation on nuclear fuel fretting," Tribology International, vol. 39, no. 10, pp. 1305-1319, 2006.

[12] H.-K. Kim, Y.-H. Lee, and K.-H. Lee, "On the geometry of the fuel rod supports concerning a fretting wear failure," Nuclear Engineering and Design, vol. 238, no. 12, pp. 3321-3330, 2008.

[13] S. J. King, M. Y. Young, D. D. Seel, M. E. Conner, R. Y. Lu, and D. V. Paramonov, "Flow Induced Vibration and Fretting Wear in PWR Fuel," pp. 639-648, 2002.

[14] M. Aulló and W. D. Rabenstein, "European Fuel Group Experience on Control Rod Insertion and Grid to Rod Fretting," in Structural Behaviour of Fuel Assemblies for Water Cooled Reactors: Proceedings of a Technical Meeting Held in Cadarache, France, 22-26 November 2004, 2005, p. 147.

[15] P. R. Rubiolo and D. V. Paramonov, "Study of fuel rod and grid supports interaction," 2003 ASME Pressure Vessels and Piping Conference, pp. 139-145, 2003.

[16] P. R. Rubiolo and M. Y. Young, "On the factors affecting the fretting-wear risk of PWR fuel assemblies," Nuclear Engineering and Design, vol. 239, no. 1, pp. 68-79, 2009.

[17] R. Y. Lu, Z. Karoutas, and T.-L. Sham, "CASL virtual reactor predictive simulation: Gridto-Rod Fretting wear," JOM, vol. 63, no. 8, pp. 53-58, 2011.

[18] A. Billerey, "Evolution of fuel rod support under irradiation-Impact on the mechanical behaviour of fuel assemblies," Structural behaviour of fuel assemblies for water cooled reactors, (in Cadarache, France, 22-26 November 2004), IAEA-TECDOC-1454, pp. 101$111,2005$.

[19] M. Griffiths, R. A. Holt, and A. Rogerson, "Microstructural aspects of accelerated deformation of Zircaloy nuclear reactor components during service," Journal of Nuclear Materials, vol. 225, pp. 245-258, 1995. 
[20] A. Patra, C. N. Tomé, and S. I. Golubov, "Crystal plasticity modeling of irradition growth in Zircaloy-2," Philosophical Magazine (under review), 2016.

[21] R. A. Lebensohn and C. N. Tomé, "A self-consistent anisotropic approach for the simulation of plastic deformation and texture development of polycrystals: application to zirconium alloys," Acta metallurgica et materialia, vol. 41, no. 9, pp. 2611-2624, 1993.

[22] A. V. Barashev, S. I. Golubov, and R. E. Stoller, "Theoretical investigation of microstructure evolution and deformation of zirconium under neutron irradiation," Journal of Nuclear Materials, vol. 461, pp. 85-94, 2015.

[23] S. I. Golubov, A. V. Barashev, and R. E. Stoller, "On the origin of radiation growth of hcp crystals," Oak Ridge National Laboratory, ORNL/TM-2011/473, Oak Ridge, TN, ORNL/TM-2011/473, 2011.

[24] B. N. Singh, M. Eldrup, S. J. Zinkle, and S. I. Golubov, "On grain-size-dependent void swelling in pure copper irradiated with fission neutrons," Philosophical Magazine A, vol. 82, no. 6, pp. 1137-1158, 2002.

[25] M. J. Norgett, M. T. Robinson, and I. M. Torrens, "A proposed method of calculating displacement dose rates," Nuclear Engineering and Design, vol. 33, no. 1, pp. 50-54, 1975.

[26] K. Ehrlich, "Irradiation creep and interrelation with swelling in austenitic stainless steels," Journal of Nuclear Materials, vol. 100, no. 1-3, pp. 149-166, 1981.

[27] S. I. Golubov, A. V. Barashev, and R. E. Stoller, "On the theory of radiation growth and creep of hexagonal close-packed crystals," in 18th International Symposium on Zirconium in the Nuclear Industry (submitted), Hilton Head, SC, 2016.

[28] B. T. Kelly and A. J. E. Foreman, "The theory of irradiation creep in reactor graphite-The dislocation pinning-unpinning model," Carbon, vol. 12, no. 2, pp. 151-158, 1974.

[29] C. N. Tomé et al., "Role of internal stresses in the transient of irradiation growth of Zircaloy-2," Journal of Nuclear Materials, vol. 227, no. 3, pp. 237-250, 1996.

[30] P. A. Turner, C. N. Tomé, N. Christodoulou, and C. H. Woo, "A self-consistent model for polycrystals undergoing simultaneous irradiation and thermal creep," Philosophical Magazine A, vol. 79, no. 10, pp. 2505-2524, 1999.

[31] P. A. Turner and C. N. Tomé, "Self-consistent modeling of visco-elastic polycrystals: Application to irradiation creep and growth," Journal of the Mechanics and Physics of Solids, vol. 41, no. 7, pp. 1191-1211, 1993.

[32] C. N. Tomé, C. B. So, and C. H. Woo, "Self-consistent calculation of steady-state creep and growth in textured zirconium," Philosophical Magazine A, vol. 67, no. 4, pp. 917-930, 1993.

[33] ABAQUS Version 6.14-1. Dassault Systémes Simulia Corp., Providence, RI, 2014.

[34] J. Segurado, R. A. Lebensohn, J. LLorca, and C. N. Tomé, "Multiscale modeling of plasticity based on embedding the viscoplastic self-consistent formulation in implicit finite elements," International Journal of Plasticity, vol. 28, no. 1, pp. 124-140, 2012.

[35] M. Knezevic et al., "Integration of self-consistent polycrystal plasticity with dislocation density based hardening laws within an implicit finite element framework: Application to low-symmetry metals," Journal of the Mechanics and Physics of Solids, vol. 61, no. 10, pp. 2034-2046, 2013.

[36] W. Liu, R. Montgomery, C. Tomé, C. Stanek, and J. Hales, "VPSC implementation in the BISON-CASL code for modeling large deformation problems," in ANS MC2015 - Joint International Conference on Mathematics and Computation $(M \& C)$, Supercomputing in 
Nuclear Applications (SNA) and the Monte Carlo (MC) Method, Nashville, TN, 2015, pp. $1-15$.

[37] R. D. McGinty, "Multiscale representation of polycrystalline inelasticity," Georgia Institute of Technology, Atlanta, USA, 2001.

[38] R. A. Holt, A. R. Causey, N. Christodoulou, M. Griffiths, E. T. C. Ho, and C. H. Woo, "Non-linear irradiation growth of cold-worked Zircaloy-2," presented at the Zirconium in the Nuclear Industry: Eleventh International Symposium, ASTM STP 1295, E.R. Bradley and G.P. Sabol, Eds., 1996, pp. 623-637.

[39] D. Lhuillier and C. Tome, "Modeling of irradiation and thermal creep of pressure tubes: 1. Texture reduction and interpolation," Atomic Energy of Canada Ltd., COG-94-232 RC1222, 1995.

[40] R. Liu, A. Prudil, W. Zhou, and P. K. Chan, "Multiphysics coupled modeling of light water reactor fuel performance," Progress in Nuclear Energy, vol. 91, pp. 38-48, Aug. 2016.

[41] R. L. Williamson, "Enhancing the ABAQUS thermomechanics code to simulate multipellet steady and transient LWR fuel rod behavior," Journal of Nuclear Materials, vol. 415, no. 1, pp. 74-83, Aug. 2011.

[42] R. L. Williamson et al., "Multidimensional multiphysics simulation of nuclear fuel behavior," Journal of Nuclear Materials, vol. 423, no. 1-3, pp. 149-163, Apr. 2012.

[43] H. Jiang, J. Qu, R. Y. Lu, and J.-A. J. Wang, "Grid-to-rod flow-induced impact study for PWR fuel in reactor," Progress in Nuclear Energy, vol. 91, pp. 355-361, 2016.

[44] F. Tinti, "Uniaxial In-Reactor Creep of Zircaloy-2: Stress, Flux, and Temperature Dependence," Nuclear Technology, vol. 60, no. 1, pp. 104-113, 1983.

[45] G. Simmons and H. Wang, Single crystal elastic constants and calculated aggregate properties. Cambridge University Press, 1971.

[46] U. F. Kocks, C. N. Tomé, and H.-R. Wenk, Texture and anisotropy: preferred orientations in polycrystals and their effect on materials properties. Cambridge University Press, 2000. 